This item was submitted to Loughborough's Research Repository by the author.

Items in Figshare are protected by copyright, with all rights reserved, unless otherwise indicated.

\title{
A deep convolutional neural network model for rapid prediction of fluvial flood inundation
}

PLEASE CITE THE PUBLISHED VERSION

https://doi.org/10.1016/j.jhydrol.2020.125481

PUBLISHER

Elsevier

VERSION

AM (Accepted Manuscript)

\section{PUBLISHER STATEMENT}

This paper was accepted for publication in the journal Journal of Hydrology and the definitive published version is available at https://doi.org/10.1016/j.jhydrol.2020.125481.

\section{LICENCE}

CC BY-NC-ND 4.0

\section{REPOSITORY RECORD}

Kabir, Syed, Sandhya Patidar, Xilin Xia, Qiuhua Liang, Jeffrey Neal, and Gareth Pender. 2020. "A Deep Convolutional Neural Network Model for Rapid Prediction of Fluvial Flood Inundation”. Loughborough University. https://hdl.handle.net/2134/12933764.v1. 


\section{A deep convolutional neural network model for rapid prediction of}

2 fluvial flood inundation

3 Syed Kabir ${ }^{1,2 *}$, Sandhya Patidar ${ }^{2}$, Xilin Xia $^{1}$, Qiuhua Liang ${ }^{1}$, Jeffrey Neal $^{3}$

4 and Gareth Pender ${ }^{2}$

$5{ }^{1}$ School of Architecture, Building and Civil Engineering, Loughborough University,

6 Loughborough, United Kingdom.

$7 \quad$ x.xia2@lboro.ac.uk; q.liang@lboro.ac.uk

$8{ }^{2}$ School of Energy, Geoscience, Infrastructure and Society, Heriot-Watt University,

9 Edinburgh, United Kingdom.

s.patidar@hw.ac.uk ; g.pender@hw.ac.uk

$11{ }^{3}$ School of Geographical Sciences, University of Bristol, Bristol, United Kingdom

12 j.neal@bristol.ac.uk

$14 *$ Corresponding author

15 Syed Kabir

16 School of Architecture, Building and Civil Engineering

17 Loughborough University

18 Loughborough, Leicestershire, LE11 3TU 


\section{Abstract}

22 Most of the two-dimensional (2D) hydraulic/hydrodynamic models are still computationally too demanding for real-time applications. In this paper, an innovative modelling approach based on a deep convolutional neural network (CNN) method is presented for rapid prediction of fluvial flood inundation. The CNN model is trained using outputs from a 2D hydraulic model (i.e. LISFLOOD-FP) to predict water depths.

27 The pre-trained model is then applied to simulate the January 2005 and December 2015 28 floods in Carlisle, UK. The CNN predictions are compared favourably with the outputs produced by LISFLOOD-FP. The performance of the CNN model is further confirmed by benchmarking against a support vector regression (SVR) method. The results show

31 that the CNN model outperforms SVR by a large margin. The CNN model is highly

32 accurate in capturing flooded cells as indicated by several quantitative assessment

33 matrices. The estimated error for predicting maximum flood depth is $0 \sim 0.2$ meters for 34 the 2005 event and $0 \sim 0.5$ meters for the 2015 event at over $99 \%$ of the cells covering

35 the computational domain. The proposed CNN method offers great potential for real36 time flood modelling/forecasting considering its simplicity, superior performance and 37 computational efficiency.

\section{Keywords}

39 Rapid flood modelling; deep learning; convolutional neural network; machine learning;

40 flood inundation

\section{Introduction}

42 Two-dimensional (2D) hydraulic/hydrodynamic models have been widely applied to

43 simulate complex hydrological processes and flood dynamics. Recent advancements in 44 computing technology along with the increasing availability of high-resolution remotely 
sensed data, such as terrain elevation and river morphology, have enabled these sophisticated models to be applied at the regional to global scales (e.g., Yamazaki et al., 2011, de Paiva et al., 2013). However, due to their high computational demand, it is still challenging to use these physically-based sophisticated models for operational real-time flood forecasting (Bhola et al., 2018).

Considerable research effort has been devoted to improving the overall performance of hydraulic/hydrodynamic models for large-scale flood modelling. For example, the computational efficiency of these models may be improved by implementing parallel computing algorithms to take advantages of multiple processors (Neal et al., 2018, Sanders \& Schubert, 2019). Xia et al. (2019) developed a new fully hydrodynamic modelling framework that utilizes the state-of-the-art high-performance graphics processing units (GPUs) for modelling fluvial flooding from rainfall-generated overland flow to inundation at a high spatial resolution across a large catchment of 2500 $\mathrm{km}^{2}$. However, despite the advances in high-performance computing technology and the development of subsequent computational methods, significant challenges still exist regarding the application of 2D hydrodynamic models for operational flood forecasting. One of the key challenges is that, while it is now possible to make a single model run in real-time using a GPU-accelerated hydrodynamic model (e.g., Ming et al., 2020), it is still computationally prohibited to run such a model multiple times using ensemble numerical weather predictions to provide reliable flood forecasts with an acceptable lead time.

An alternative approach is to adopt an offline method for operational flood inundation mapping as proposed in Bhola et al. (2018). The proposed offline method requires the construction of a database using pre-run inundation maps and river discharges. During or before a flood event, the inundation maps with a matching 
discharge are requested and retrieved from the database to provide forecasts (Leedal et al., 2010, Bhola et al., 2018). Although such a system does not require live 2D simulations, preparing the database of inundation maps can be labour intensive and requires storage of a large volume of data. Furthermore, due to changing environment, e.g., land-use change, geomorphological change and engineering construction, the flood scenarios may become outdated and new simulations are required to regularly update the database, creating extra effort and resources for maintenance. The ideal solution to these various technical challenges would be to develop a new modelling approach that can relax the mentioned computational burden while still being able to generate practically useful and statistically significant information to support real-time flood forecasting. A possible solution would be to use machine

81 learning (ML) models that can closely emulate the outputs of 2D hydrodynamic models. 82 Application of ML techniques for rainfall-runoff forecasting has been investigated for a few decades. In contrast, research on the application of ML for flood inundation modelling remains very limited and only a handful of such ML approaches have been reported to date. For example, in Chang et al. (2010, 2014) and Shen \& Chang (2013), hybrid ML techniques were successfully used for flood modelling. Chang et al. (2018a) developed a self-organizing map (SOM), an artificial neural network (ANN) designed for clustering operations, integrated with recurrent nonlinear autoregressive exogenous (R-NARX) networks for flood modelling at regional scale, giving flood forecasting of 90 up to $12 \mathrm{~h}$ ahead in the Kemaman River Basin, Malaysia. This approach was further 91 improved in Chang et al. (2018b) by developing an intelligent hydroinformatics integration platform (IHIP) to provide a user-friendly web interface for enhanced capability in online flood forecasting and risk management. Of particular relevance to

94 the current study, Liu \& Pender (2015) developed an inundation model based on a 
support vector regression (SVR) algorithm for emulating the outputs of a fine grid model (FGM). In their approach, SVRs were first trained using a small number of the outputs from the FGM and then applied to predict water depths and velocities at the target locations. Bermúdez et al. (2019) presented a least squared-support vector machine (LS-SVM) method to compute the spatial distribution of the maximum water depth and velocity in a coastal urban area using three sets of flow and tidal data.

Berkhahn et al. (2019) proposed an ensemble neural network method to predict in realtime the maximum water levels of a flash flood event induced by spatially uniform rainfall. A data-driven $3 \mathrm{~h}$ ahead fluvial flood inundation mapping framework was proposed by Kabir et al. (2020), which shows that the wet/dry condition of the cells inside the simulation domain is a function of the upstream discharge magnitude and duration. However, this study only focused on classifying wet/dry cells to indicate flooded areas in a small English town of $\sim 5 \mathrm{~km}^{2}$ and the flooding processes was only driven by one river channel. These studies have demonstrated that the computationally much less expensive ML approaches may be used to efficiently predict flood variables once they are trained and calibrated appropriately.

When selecting an ML technique for flood modelling, we should bear in mind that most of the existing algorithms are not suitable for multi-output scenarios, i.e. predicting a flood variable (e.g., depth) in multiple cells through a single model. In this regard, artificial neural networks (ANNs) may potentially be more useful as it can be implemented to solve both of the single-output and multi-output problems. Over the last decade, convolution neural networks (CNNs) have gained unprecedented success in solving computer vision problems due to their ability to extract unknown features and learn compact representations and represent the current leading deep learning (DL) 
119 paradigm. Nevertheless, the potentiality of CNNs in modelling high-resolution flood

120 inundation has not yet been tested and confirmed.

121 In this work, we present an innovative rapid fluvial flood modelling approach

122 based on CNNs to predict spatially distributed water depths for two large flood events

123 in the City of Carlisle, UK. This is for the first time that the capability of a one-

124 dimensional CNN (1D-CNN) model, accelerated by modern GPUs, is investigated and

125 tested for rapid prediction of fluvial flooding in a domain containing over half of a

126 million cells. The rest of the paper is organised as follows: Section 2 introduces

127 methodology including the proposed CNN modelling framework; the experimental

128 setup and application are explained in Section 3; Section 4 presents and discusses the

129 results; and finally, brief conclusions are drawn in Section 5.

\section{Methodology}

131 This section explains how the CNN-based fluvial flood modelling framework is

132 developed for reproducing two historical flood events in the City of Carlisle, UK.

\subsection{Overall research strategy}

134 Fig. 1 illustrates the five steps in developing and assessing the new CNN model for

135 fluvial flood inundation modelling:

136 Step 1: Generate sufficient number of synthetic hydrographs to represent different

137 hydrological and flood conditions at the upstream boundary points of the three river

138 channels feeding to the case study site;

139 Step 2: Use these hydrographs as the boundary conditions to drive a 2D-

140 hydraulic/hydrodynamic model (e.g. LISFLOOD-FP as adopted in this study) to create

141 spatially distributed inundation sequences and subsequently derive the time-series of 
142 water depth at each cell in the domain for the entire flood duration;

143 Step 3: Generate training and validation datasets for the candidate DL model (e.g. CNN

144 model in this work); for training, the upstream synthetic hydrographs are used as input

145 variables and the hydraulic simulation outputs (i.e. water depth sequences) are used as

146 the target variable;

147 Step 4: Develop, train and optimize the candidate DL model;

148 Step 5: Use observed or predicted upstream hydrographs to drive the trained DL model 149 to predict fluvial flood inundation (e.g. to reproduce the Carlisle 2005 and 2015 floods 150 in this work) and assess its performance.

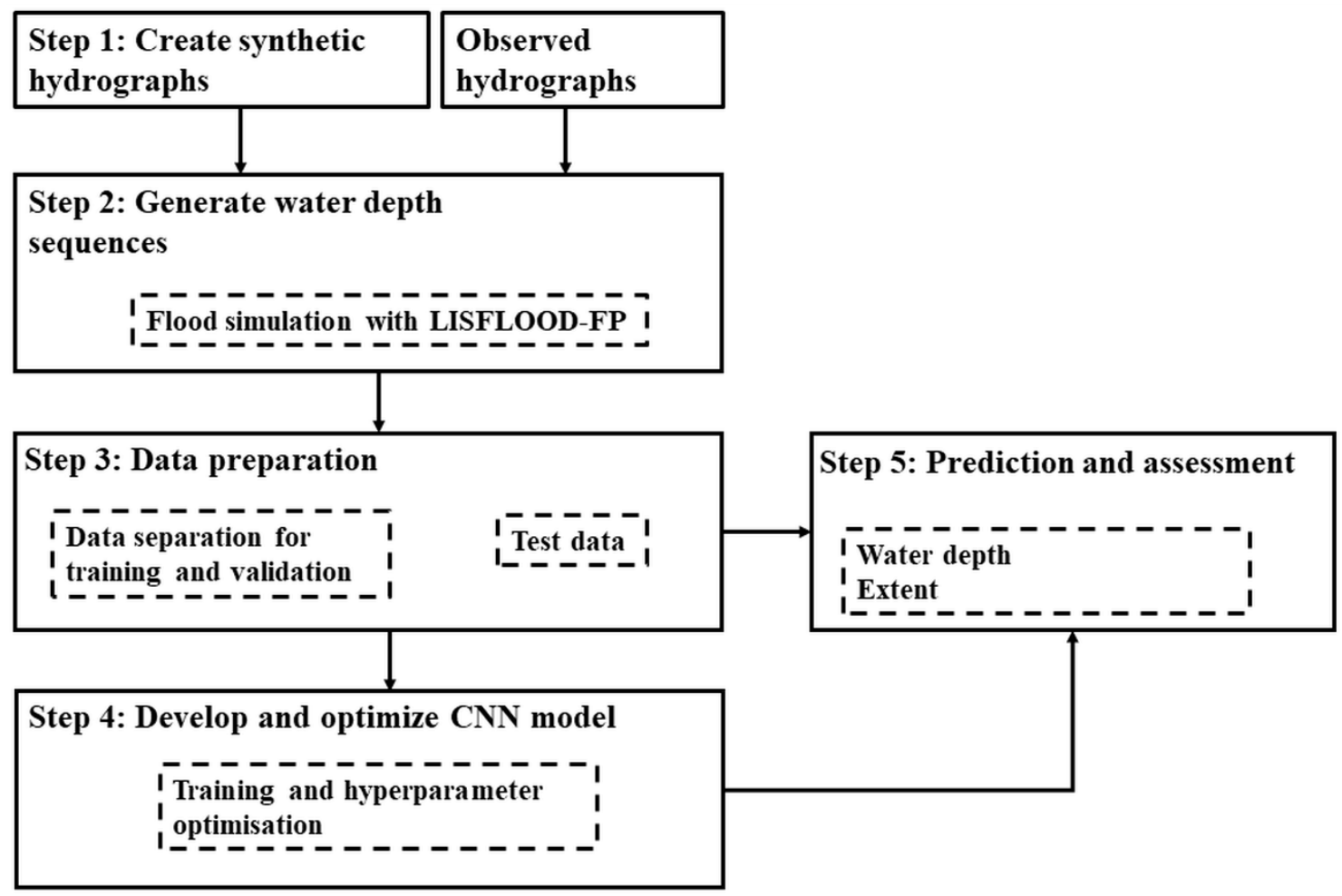

152 Figure 1. The core steps in developing and testing the proposed CNN flood model.

To assess the capability of the proposed CNN model in emulating the results of 
compared with the outputs from the adopted hydraulic model, i.e. LISFLOOD-FP. In

157 addition, the performance of the $\mathrm{CNN}$ model will be further evaluated by comparing

158 with an SVR approach, one of the popular ML methods used in earlier studies (e.g., Lin

159 et al., 2013, Liu \& Pender, 2015, Jhong et al., 2018, Bermúdez et al., 2019). A point-

160 wise comparison is made between the models at 18 pre-selected control points (13 of

161 which are taken from the list of locations where water depths were surveyed by the EA

162 and University of Bristol after the 2005 event) that represent the main flood risk zone.

163 Several assessment matrices are adopted here to quantify the prediction errors.

164 The root mean squared error (RMSE) (Barnston, 1992) is defined as

$$
R M S E=\sqrt{\frac{\sum_{i=1}^{N}\left(O_{i}-P_{i}\right)^{2}}{N}}
$$

166 where $N$ is the sample size, $O_{i}$ and $P_{i}$ are the 'observed' and 'predicted' values, respectively. $R M S E=0$ returns a perfect fit between the predicted and 'observed' data. be calculated as follows

$$
N S E=1-\frac{\sum_{i=1}^{N}\left(O_{i}-P_{i}\right)^{2}}{\sum_{i=1}^{N}\left(O_{i}-\bar{o}\right)^{2}}
$$

where $\bar{o}$ is the mean of 'observed' data. The value of NSE generally varies between 0 and 1 , where $N S E=1$ represents a perfect fit between the reference and predicted data and a negative $N S E$ indicates that the model fails to reproduce the test case.

To evaluate the accuracy in predicting flood inundation, the precision, recall and F1 metrics (Eq. 3-5) are also considered to demonstrate how precisely the models predict 'true positives' (cells correctly predicted as flooded by the predictive models) to the total predicted positives (the sum of the cells correctly and wrongly predicted as 
178 flooded) and to the total actual positives (cells predicted as flooded by the LISFLOOD-

179 FP) (Bermúdez et al., 2019) over the entire domain:

$$
\text { Precision }=\frac{\text { True positive }}{\text { Total predicted positive }}
$$

$$
\text { Recall }=\frac{\text { True positive }}{\text { Total actual positive }}
$$

$$
F 1=2 \times \frac{\text { Precision } \times \text { Recall }}{\text { Precision }+ \text { Recall }}
$$

183 A higher precision value implies that most of the cells predicted as flooded by the predictive models are also classified as flooded by the reference model, i.e. LISFLOOD-FP. A higher recall value means that cells classified as flooded are well captured by the predictive models. $F 1$ is the harmonic mean of recall and precision

187 metrics and defines how well the model predictions match the reference results, with a 188 score of 1 returning a perfect match. capability of the CNN model in emulating the outputs of a 2D hydrodynamic/hydraulic

191 model. Therefore, the outputs of the LISFLOOD-FP model rather than the field

192 observations are used as the reference to assess the predictive performances of the ML

193 models under consideration.

\subsection{Overview of models being used}

195 In this section we describe the hydraulic and the ML models adopted to develop, train

196 and assess the proposed rapid flood modelling system.

\subsubsection{Convolutional Neural Networks (CNNs)}

198 Since its inception in 1990, CNNs have become a research hotspot and the de facto 
standard for various ML and computer vision (CV) applications. In particular, the

200 annual ImageNet large scale visual recognition challenge (ILSVRC) 2012 has changed

201 the course of image classification problems through the application of deep CNNs.

202 However, the key factor which has made the deep CNNs extremely popular is the ever-

203 increasing computational power of the modern computers.

204 CNNs are, in general, feed-forward neural networks with alternating

205 convolutional and subsampling layers and are predominantly trained in a supervised

206 manner (Kiranyaz et al., 2019). Deep CNNs have been exclusively developed to operate

207 on 2D data (images and videos) and commonly known as '2D-CNNs'. 2D-CNNs can

208 extract features and learn complex objects from large volume of labelled data. Whilst

209 the classical CNNs were developed specifically for 2D signals, Kiranyaz et al. (2015)

210 proposed the first 1D-CNN to handle sequential data (1D signal). Since then, 1D-CNNs

211 have gained significant popularity and been successfully applied in various fields, e.g.,

212 biomedical data classification (Zihlmann et al., 2017), structural damage detection

213 (Abdeljaber et al., 2018) and sentiment analysis (Munandar et al., 2018).

\subsubsection{LISFLOOD-FP flood inundation model}

215 The physically-based LISFLOOD-FP hydraulic model is used to generate training

216 samples for the data-driven predictive models considered in this work. First reported by

217 Bates \& De Roo (2000), LISFLOOD-FP is a raster-based model for simulating fluvial

218 or coastal flood inundation. The model has been improved significantly over the last

219 two decades and tested successfully in numerous case studies across the globe (e.g.,

220 Knijff et al., 2010, Amarnath et al., 2015, Komi et al., 2017).

221 LISFLOOD-FP solves the zero-inertial approximation of the Saint Venant

222 equations (i.e. diffusion-wave approximation) using an explicit forward difference 
223 scheme on a staggered grid over a 2D plane. The technical details of the model can be

224 found in Bates \& De Roo (2000). Specific to the present study, the LISFLOOD-FP

225 version 6.3.1 is used, which was implemented with an 'acceleration' solver to recover

226 the inertial terms from the Saint Venant equations (Bates et al., 2010, de Almeida et al.,

227 2012) for calculating floodplain inundation and a 'sub-grid' solver (Neal et al., 2012)

228 for representing channelized flows below the adopted grid resolution. The model and

229 relevant documentation can be acquired from

230 http://www.bristol.ac.uk/geography/research/hydrology/models/lisflood/.

232 The SVR is a kernel-based supervised learning method developed from the original

233 support vector machine (SVM) method (Cortes \& Vapnik, 1995) to solve regression

234 problems. The basic feature of SVR is to map the input space into a high-dimensional

235 feature space using a non-linear mapping function (for solving non-linear problems),

236 through which the non-linearity of input vectors becomes linearly separable

237 (Raghavendra and Deka, 2014).

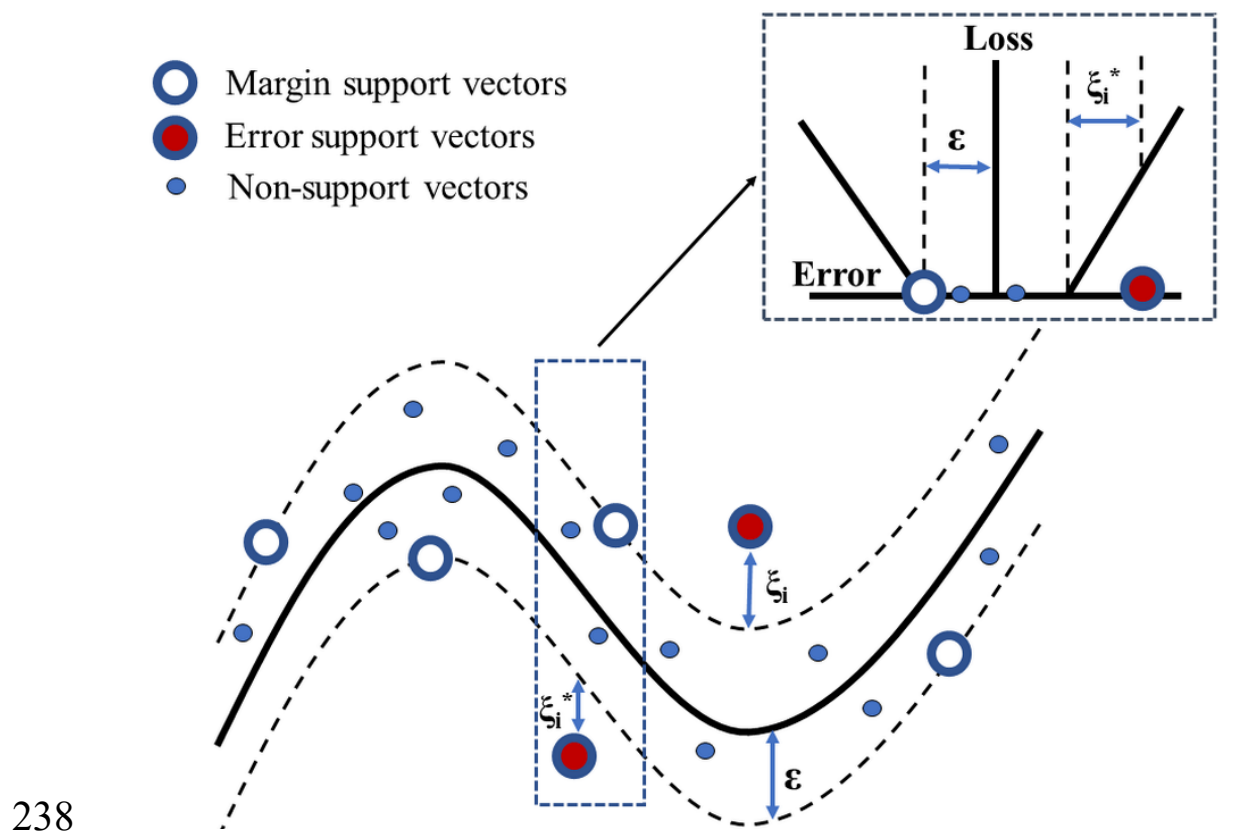


Figure 2. Non-linear SVR with $\varepsilon$-insensitive zone in the feature space.

The concept of non-linear SVR in the feature space is illustrated in Figure 2, in

242 which $\xi_{i}$ and $\xi_{i}{ }^{*}$ are the so-called slack variables and these non-negative variables are

243 introduced to estimate the deviation of training data samples lying outside of the $\varepsilon$ -

244 insensitive zone. The effectiveness of an SVR model depends upon several mutually

245 linked parameters, e.g., kernel parameters, cost parameter and the width of the $\varepsilon$ -

246 insensitive zone. The cost parameter is a positive constant indicating the smoothness of

247 the approximation function. A large cost parameter may overfit the training data but an

248 inappropriately small value may lead to underfitting. Parameter $\varepsilon$ controls the width of

249 the insensitive zone, influencing the number of support vectors and thus the overall

250 generalization ability of an SVR model.

251 In this study, to draw a fair comparison between the CNN and SVR flood

252 models, identical training and testing datasets are used. The same parameter

253 optimisation technique is also employed to search for appropriate model

254 hyperparameters.

\section{Model setup and application}

256 This section gives the key details related to how LISFLOOD-FP, CNN and SVM

257 models are setup and applied, and the data being used.

\subsection{Case study}

259 To demonstrate the performance of the CNN flood model, the city of Carlisle is used as

260 the case study in this work and the site-specific information and key hydrometric and 261 spatial data are introduced herein. 
263 Located in the downstream Eden Catchment in the Northwest England, the city of

264 Carlisle is highly prone to flooding. The total drainage area of Eden Catchment is nearly $2500 \mathrm{~km}^{2}$ and is fed by an average precipitation of approximately $1148 \mathrm{~mm} / \mathrm{yr}$ (Allen et al., 2010). The study domain covers about $14.5 \mathrm{~km}^{2}$ of the urbanised area of Carlisle (Fig. 3), which has been heavily hit by historical events including the 2005 and 2015 floods. There are three main river channels within the area of interest, i.e. River Eden,

269 Petteril and Caldew, which drive the flood dynamics. The locations (within the study domain) at high risk of flooding are at the confluence of the Rivers Eden and Caldew at Willow Holme, and the confluences of Little Caldew, River Petteril and River Eden at Durranhill, Botcherby and Warwick Road. In 2005, the River Eden and its two tributaries (River Caldew and River Petteril) caused unprecedented flooding on the $8^{\text {th }}$ January due to persistent rainfall that started on the $7^{\text {th }}$ January (Roberts et al., 2009). The onset of the event was slow with initial flooding occurring in the early hours of the day, which was well before the peak arrived around noon. The inundation predominantly occurred in the residential/commercial zones along the channels and low-lying rural areas situated on the north-eastern part of the city. The annual exceedance probability (AEP) of the event was estimated to be 0.59\%, corresponding to a 170-year return period (Cumbria County Council, 2017).

281 Following this flood event which affected approximately 1600 properties, flood defences were constructed to protect the high-risk areas of the city for flood events

283 similar to or greater than the one in 2005 (AEP 0.5\%). However, on $5^{\text {th }}$ and $6^{\text {th }}$

284 December 2015, the Storm Desmond brought in record-breaking rainfall that 285 subsequently led to widespread flooding across the Eden Catchment, with Carlisle again 286 being heavily hit. The magnitude of the event (AEP $0.33 \%$ ) exceeded the designed 
287 criteria of the Carlisle flood defence scheme (Cumbria County Council, 2017). The

288 flooding followed by overtopping of the embankments and flood walls devastated the

289 city and its people, affecting approximately 2100 properties. The flow rate recorded at

290 the Sheepmount gauging station in River Eden in the morning of the $6^{\text {th }}$ December was

$2911680 \mathrm{~m}^{3} / \mathrm{s}$, breaking any records on book. These two major events provide an

292 opportunity to test whether a model performs consistently under different conditions,

293 i.e. with and without flood defences. For this reason, both of the 2005 and 2015 flood

294 events are used to test the proposed CNN model.

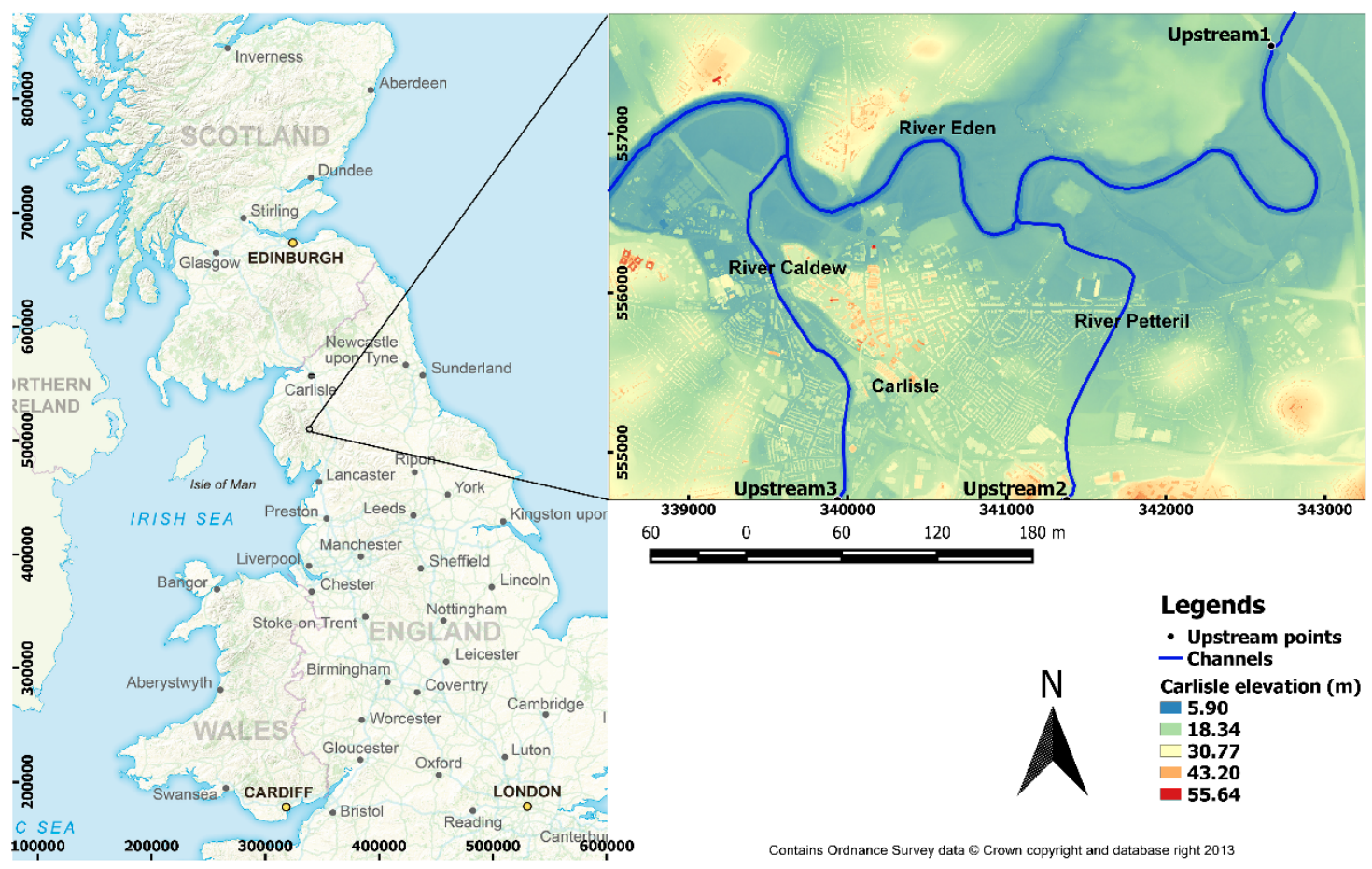

296 Figure 3. Study domain and topography.

\subsubsection{Hydrometric and topographic data}

298 Hydrometric and topographic data are required to set up and run LISFLOOD-FP for

299 predicting flood inundation. Essential data to reproduce the Carlisle flood events

300 include upstream boundary conditions (e.g. flow hydrographs) and digital elevation

301 model (DEM) for the case study site. In this work, we use the DEM provided by the 
302 School of Geographical Sciences at the University of Bristol, which was created by

303 further processing the LiDAR DEM provided by the UK Environment Agency (EA) by

304 removing the noises and obstacles (e.g. trees and bridges). The DEM has a $5 \mathrm{~m}$ spatial

305 resolution and was deemed sufficient to reproduce the flood dynamics using

306 LISFLOOD-FP in the previous studies (e.g. Neal et al., 2013). But this DEM is only

307 valid for the 2005 flood event because new flood defences have been built afterwards.

308 To model the 2015 event and investigate the impact of flood alleviation scheme, the

309 data in the format of a shapefile are acquired from the government open data platform

310 (https://data.gov.uk/) for the defences built between 2005 and 2015 and then embedded

311 in the DEM for reproducing the 2005 event, in which all of the flood defences are

312 assigned with a constant height of $2 \mathrm{~m}$.

313 To drive the two flood events, boundary conditions are needed at the upstream

314 points of the three feeding rivers. The three boundary points are marked in Fig. 3 as

315 "Upstream 1" (Easting: 342682, Northing: 557532) located under the M6 bridge in the

316 North-East of the domain, and "Upstream 2" (Easting: 341362, Northing: 554702) and

317 "Upstream 3" (Easting: 339947, Northing: 554702) located at the south edge of the

318 domain. Herein, the coordinate reference system OSGB 1936 (EPSG:27700) is adopted.

319 It is worth noting that these upstream boundary points do not coincide with flow

320 observation gauges in the rivers and therefore no direct observation data are available to

321 drive the flood simulations. For the 2005 event, 15-minute discharge hydrographs

322 obtained through 1D flood routing are available from previous studies (e.g. Neal et al.,

323 2013, Parkes et al., 2013) and are used in this study without further modification. For

324 the 2015 event, the 15-minute measured hydrographs from the nearby gauging stations,

325 i.e. Great Corby in Eden River, Harraby Green Business Park in Petteril and 
326 Cummersdale in Caldew are directly imposed in the respective boundary points to drive

327 the flood simulations.

\subsection{Generating input hydrographs}

329 To reproduce the 2005 and 2015 Carlisle floods and demonstrate the performance of the

330 ML-based predictive models, it is necessary to generate sufficient input (discharge) and

331 output (water depth) data to train the ML models. For this purpose, 24 'synthetic'

332 hydrographs ( 8 for each of the three upstream boundary locations) with various peaks

333 and durations are produced to represent flood scenarios of different magnitudes. In each

334 of the upstream locations, hydrographs of the historical floods with smaller peaks than

335 the January 2005 event are selected and adjusted using the following formula to

336 increase their magnitudes as appropriate:

$$
Q_{n}=Q_{o b s} \times \frac{P_{e a k_{\max }}}{Q_{\max }} \quad\left\{\text { Peak }_{\max }>\text { Max } Q_{o b s}\right\}
$$

338 where $Q_{n}$ is the synthetic flow discharge, $P e a k_{\max }$ is the user specified peak, $Q_{o b s}$ is

339 the observed discharge and $Q_{\max }$ is the observed peak discharge. This generates the

340 synthetic hydrographs A-E as shown in Figure 4. In addition, three more hydrographs

341 (hydrographs F-H in Figure 4) are selected from the historical records at different

342 gauging stations of River Eden to complete the training datasets (for all three upstream

343 points). When generating or selecting the hydrographs, the flow in the River Eden is

344 ensured to be substantially larger than the flows in the two tributaries to reflect the

345 reality. The final $8 \times 3$ 'synthetic' hydrographs are presented in Figure 4, together with

346 the routed/measured hydrographs to drive the 2005 and 2015 flood simulations. A brief

347 summary of these hydrographs is provided in Table 1. 

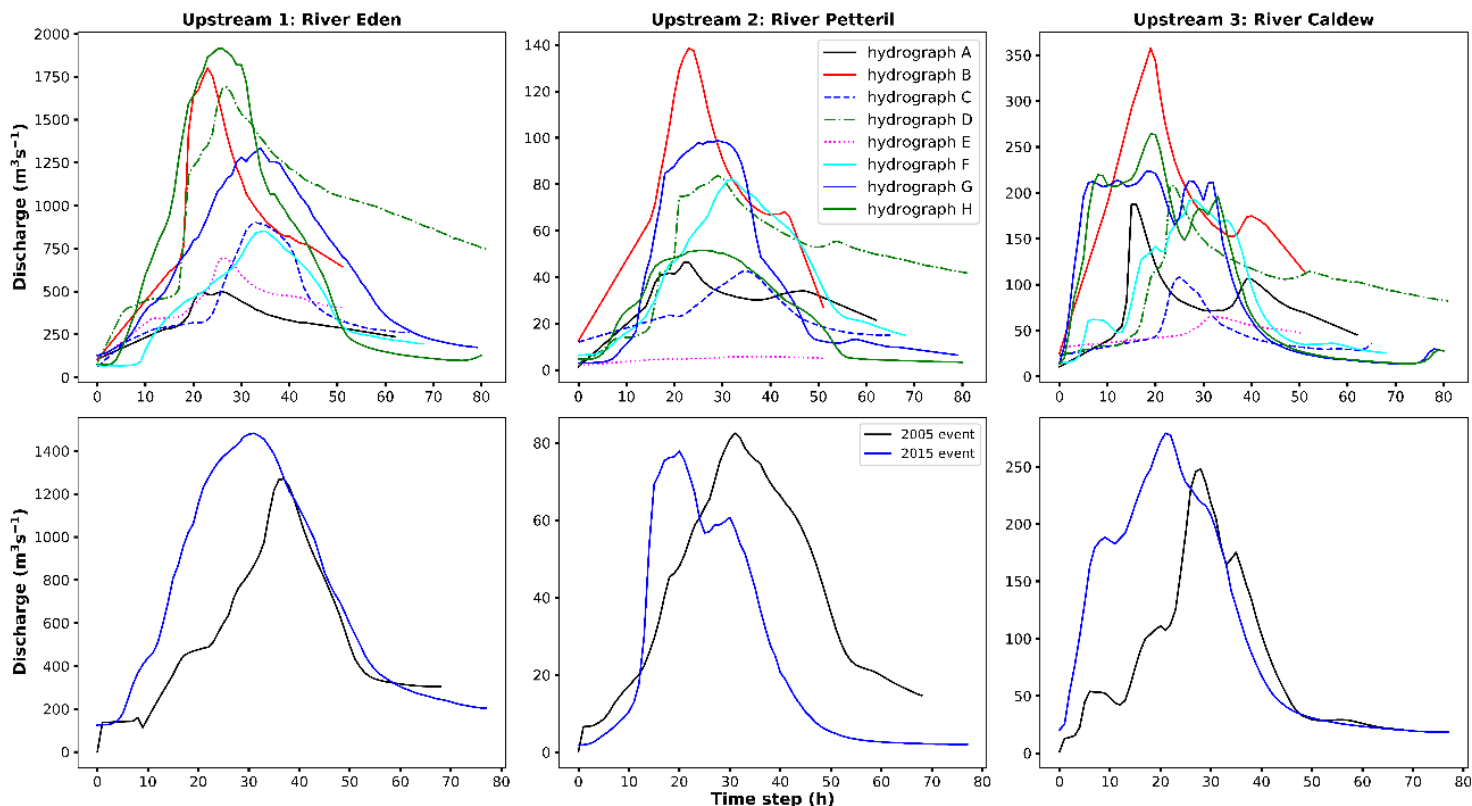

349 Figure 4. The 'synthetic' and routed/observed hydrographs used to train and test the ML

350 predictive models. For the 2005 Carlisle flood event, the hydrographs start at 00:00

351 hours on $7^{\text {th }}$ January 2005 as time 0 . The hydrographs for the 2015 event start at 23.15

352 hours on $4^{\text {th }}$ December as time 0.

353 Table 1. Summary of the hydrographs used in this study.

\begin{tabular}{clcccc}
\hline \multicolumn{1}{c}{ Name } & Type & Purpose & $\begin{array}{c}\text { Upstream 1 } \\
\text { peak }\left[\mathbf{m}^{3} / \mathbf{s}\right]\end{array}$ & $\begin{array}{c}\text { Upstream 2 } \\
\text { peak }\left[\mathbf{m}^{3} / \mathbf{s}\right]\end{array}$ & $\begin{array}{c}\text { Upstream 3 } \\
\text { peak }\left[\mathbf{m}^{3} / \mathbf{s}\right]\end{array}$ \\
\hline Hydrograph A & Synthetic & Training & 500.00 & 46.81 & 194.73 \\
Hydrograph B & Synthetic & Training & 1800.00 & 138.88 & 365.65 \\
Hydrograph C & Synthetic & Training & 900.00 & 42.59 & 109.20 \\
Hydrograph D & Synthetic & Training & 1700.00 & 83.68 & 209.63 \\
Hydrograph E & Synthetic & Training & 700.00 & 5.74 & 65.43 \\
Hydrograph F & Synthetic & Training & 854.28 & 82.57 & 193.33 \\
Hydrograph G & Synthetic & Training & 1338.07 & 99.16 & 226.74 \\
Hydrograph H & Synthetic & Training & 1950.50 & 51.99 & 266.91 \\
Event 2005 & Approximated & Testing & 1272.94 & 82.57 & 248.74 \\
Event 2015 & Approximated & Testing & 1486.19 & 78.90 & 279.30 \\
\hline
\end{tabular}

354

356 To generate training samples, LISFLOOD-FP is run sixteen times using the eight sets of 
357

358

359

360

361

362

363

364

365

366

367

368

369

370

371

372

373

374

375

376

377

378

379

synthetic inflow hydrographs to produce different flood conditions in the study site described by DEMs with and without embedding the flood defences constructed after 2005. The output files contain grid-based (raster format) water depths distributing over the study site at 15-minute temporal resolution. The test samples are generated respectively using the 2005 routed hydrographs on the pre-flood defence DEM and the 2015 approximate hydrographs on the post-flood defence DEM. For all of the simulations, the friction coefficient (Manning's $n$ ) is set to be uniform across the whole domain. Three different values $\left(0.035 \mathrm{sm}^{-1 / 3}, 0.045 \mathrm{sm}^{-1 / 3}\right.$ and $\left.0.055 \mathrm{sm}^{-1 / 3}\right)$ are tested, and $0.055 \mathrm{sm}^{-1 / 3}$ produces reasonable results and hence is adopted to support the simulations to generate the training and test datasets.

Herein, a depth threshold of $0.3 \mathrm{~m}$ is applied to negate the insignificant depths from the target data, i.e.

$$
Y=\left\{\begin{array}{lr}
x & x>0.3 \\
0 & \text { Otherwise }
\end{array}\right.
$$

where $Y$ represents the water depth value in a stacked array (further introduce in Section 3.6). This value is selected based on the flood hazard thresholds for different objects (e.g. $0.15 \mathrm{~m}$ for transport links and $0.3 \mathrm{~m}$ for buildings) as suggested by Aldridge et al. (2016) when building their impact library for flood risk assessment in the UK.

\subsection{Constructing the predictive models}

In this work, the proposed CNN model is developed in Python programming language using Keras module within the Tensorflow 2.1 framework. More specifically, the sequential application programming interface (Keras Sequential API) is used to build the model, layer-by-layer. The core structure of the 1D-CNN model is graphically illustrated in Fig. 5. The network has five hidden layers, including two convolutional 
layers and three dense layers. The dense layers are fully connected and act like a multi-

381 layer perceptron (MLP) network. The output layer contains nodes equal to the number

382 of cells in the simulation domain (i.e. 581,061 for the current case study), and the input

383 layer receives the upstream flow discharge values.

384

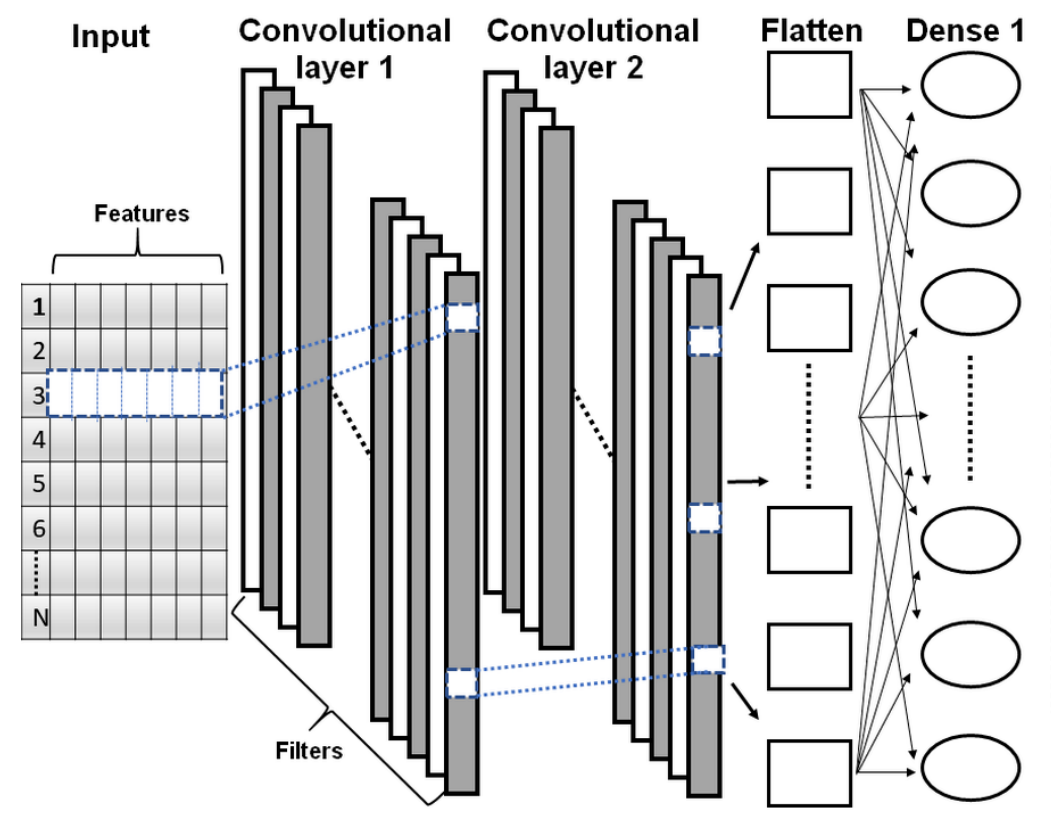

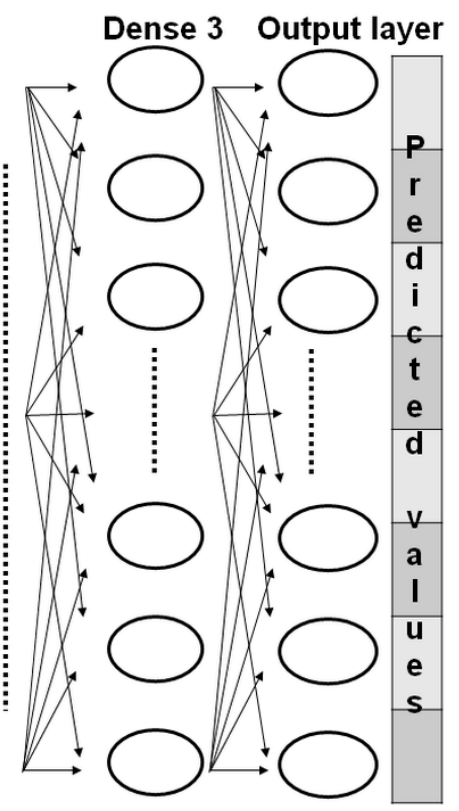

385

386

387

388

389

390

391

392

Figure 5. Structure of the adopted CNN for water depth prediction. Here, the 'features' contain the model inputs (i.e. time-series of discharge with lags and time), with each of rows $(1-\mathrm{N})$ containing information at one 'time-step'. The output of the model is an array of water depths which are then converted to spatially distributed flood maps.

In addition to the CNN, an SVR flood modelling method is also considered and constructed herein for comparison. Unlike CNN, SVR does not allow training and making predictions for all of the cells at one time and it is also not practical to calibrate an SVR model at each of the cells. Bermúdez et al. (2019) trained 25,000 individual SVR models and then linearly interpolated the predicted results to produce the distributed water depth maps across the whole simulation domain represented by a DEM of 1m spatial resolution. The area of the current study site is similar to that of 
397 Bermúdez et al. (2019) but the spatial resolution of the adopted DEM is 5m. Therefore,

398500 locations are randomly selected and spatially distributed to represent the study

399 domain, with each of these locations fitted by a separate SVR model. The regression

400 kriging (RK) interpolation method (Hengl et al., 2007) is then used to deduce the depths

401 at the unsampled locations. The SVR models are built using the Scikit-learn library in

402 Python. More details on parameter selection and optimisation are provided in the

403 following sub-sections.

\subsection{Hyperparameter optimisation}

405 The objective of hyperparameter optimisation is to find the 'best' set of parameters of a 406 given model that lead to the optimum performance as measured on the validation data.

407 In this study, the Bayesian optimisation approach is adopted to tune each of the

408 predictive models. For either of the 2005 or 2015 events (simulated with different

409 DEMs), we use the eight synthetic flood scenarios for the hyperparameter optimisation

410 and keep the 'real-event' data for model testing. We also optimise 18 SVR models for

411 each of the control points, leading to 18 sets of SVR parameters. The optimised SVR

412 models are then iteratively run using these parameter sets to find the global parameters

413 by identifying the parameter set that produces the lowest error for all 18 control points.

414 The use of a global parameter set is essential because optimising 500 SVR models is

415 practically unfeasible. The final searched hyperparameter sets of the CNN and SVR

416 models for the current case study are summarised in Table 2.

417 Table 2. Hyperparameters of the CNN and SVR predictive models.

\begin{tabular}{lllll}
\hline Model & Filter size & Neurons & Optimiser & Batch size \\
\hline CNN & {$[32,128]$} & {$[32,256,512]$} & Adam & 10 \\
\hline
\end{tabular}




\begin{tabular}{|c|c|c|c|c|}
\hline & Kernel & Cost & Epsilon & Gamma \\
\hline SVR & Radial basis & 25.296 & 0.031 & 0.016 \\
\hline
\end{tabular}

420 On each of the two DEMs for the study site, the CNN and the benchmark SVR models

421 are trained using input (predictor) and output (target) variables from the eight synthetic

422 flood scenarios (hydrographs A-H in Fig. 4). The training process is completed in two 423 steps.

424 In the first step, the input and target variables are defined by converting the eight 425 synthetic hydrographs at each of the upstream points and the corresponding raster-based flood depths predicted by LISFLOOD-FP into matrices. In this study, we explicitly use discharge values with eight antecedent time-steps (corresponds to $2 \mathrm{~h}$ of LISFLOOD-FP model initialisation time) for each of the upstream locations and the corresponding observation time as the primary inputs for predicting water depths. This leads to a total

430 of 28 input variables. The discharge with antecedent values and corresponding observation times for all eight synthetic hydrographs are stacked vertically to create the input feature matrix containing 2104 samples (Figure 6A). The target matrix is created

433 by converting the sequential water depth raster files into arrays and vertically stacking 434 them (Figure 6B). This results in a matrix of size $2104 \mathrm{X} 581061$, where 581061 is the 435 total number of cells in the domain.

436 In the second step, the ML models are trained using the input and target 437 variables. Each row in the input and output matrices is treated as a sample of the 438 training dataset. During the $\mathrm{CNN}$ training process, different regularisation techniques, 
439 including 'early stopping' (stops the training process when the model performance does

440 not improve after a certain number of iterations), 'batch normalisation' (Ioffe \&

441 Szegedy, 2015) and 'dropout' (Srivastava et al., 2014) are applied to prevent the model

442 from overfitting. It is found that, along with 'early stopping' criteria, 'batch

443 normalisation' and 'dropout' (with dropout rate $=0.2$ ) are particularly effective for

444 reproducing the 2015 event for which the flood defences are considered in the DEM.

445 Therefore, these regularisation techniques are used for the 2015 event. However,

446 inclusion of 'batch normalisation' and 'dropout' did not increase the accuracy for 2005

447 event, and therefore, only 'early stopping' criteria is applied. The training process is

448 repeated twice using respectively the 2005 and 2015 training datasets (referred to as

449 CNN-2005 and CNN-2015 models hereafter). Figure 6C sketches the training process

450 for CNN-2015. The CNN hyperparameters presented in Table 2 is slightly adjusted for

451 the $\mathrm{CNN}-2015$ by increasing the batch size to 32 . This is because, a general practice is

452 to use a larger 'batch size' when 'batch normalisation' regularisation technique is

453 applied. 


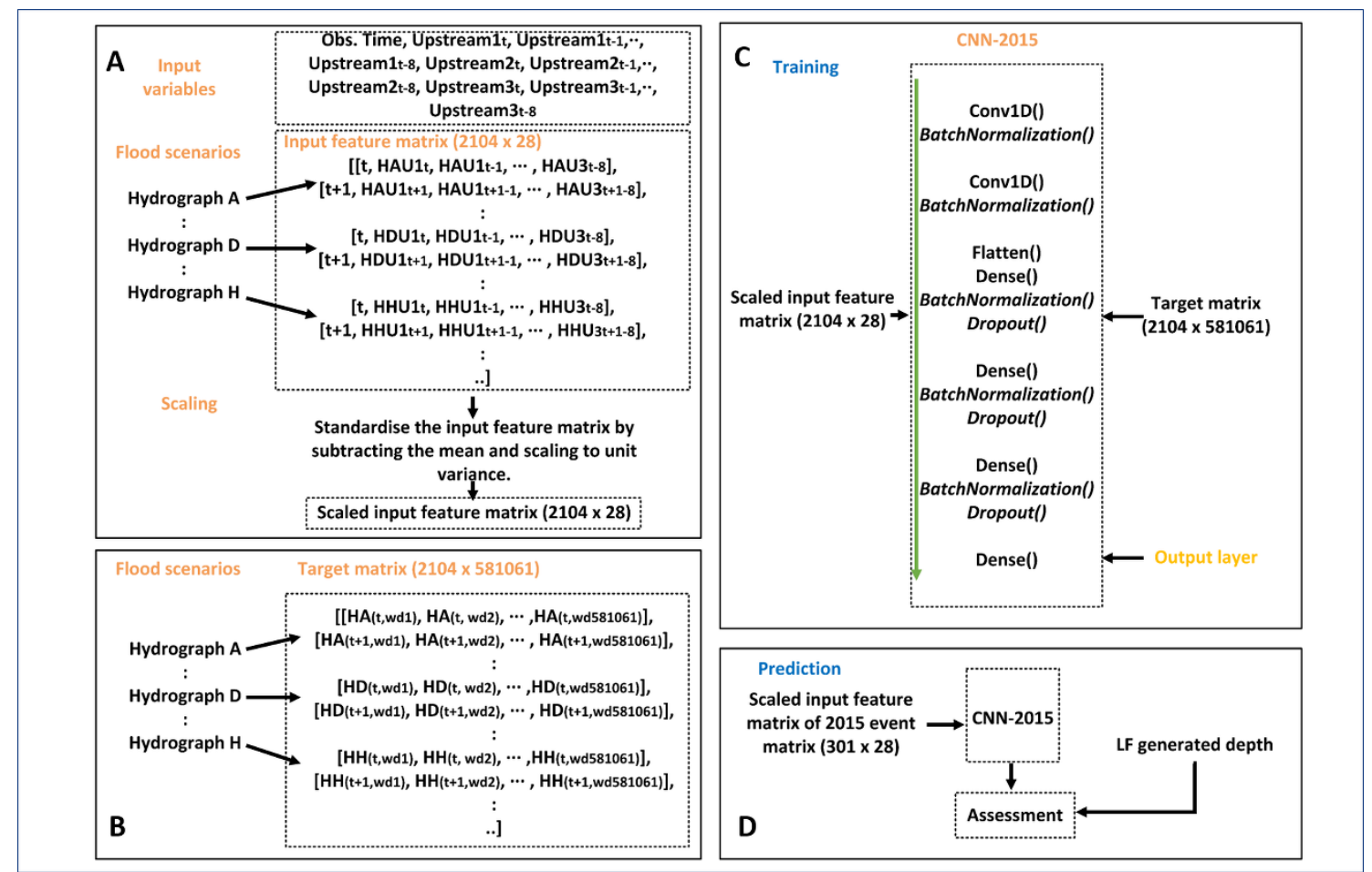

455 Figure 6. The proposed CNN inundation modelling framework. Here, for example, $t$ is

456 the flow 'observation' time, $H A U 1_{t}$ and $H A_{(t, w d l)}$ are the corresponding flow rate at

457 Upstream1 and water depth at cell number 1 for the Hydrograph A and the associated

458 flooding scenario. The 2015 flood event has 301 discharge values (301 samples) and the

4592005 event has 266 samples. The predicted outputs are compared with the LISFLOOD-

460 FP (LF) generated results.

At this stage, the SVR models are also trained using the same set of input and sampled locations (500 in total). The global model parameters given in Table 2 are used during the SVR training process. 
domain at a 15-minute time interval. The point-wise predictions from the SVR models

472 are spatially interpolated using the RK method, as mentioned in Sect. 3.4. The results

473 and performance of the models are evaluated using the assessment matrices introduced 474 previously.

\section{Results and discussion}

476

477

480

481

482

483

484

485

486

487

488

489

490

491

492

To demonstrate the performance of the proposed CNN method, the 2005 and 2015

Carlisle floods are reproduced, and the results are compared with those produced by the LISFLOOD-FP and the SVR approach in this section.

\subsection{Point-wise comparison between the CNN-2005, SVR and LISFLOOD-} FP predictions for the 2005 event

For the 2005 event, the three routed hydrographs as presented in Fig. 4 are imposed at the three upstream boundary points of the corresponding rivers to drive the three flood models under consideration. The CNN-2005 and SVR predictions in terms of timeseries of flood depth are first compared with those predicted by LISFLOOD-FP at 18 control locations as indicated in Fig. 7. The results are plotted in Fig. 8, in which a threshold of $0.3 \mathrm{~m}$ is used to screen the depth values, i.e. the predicted depth is truncated to be 0 when it is smaller than $0.3 \mathrm{~m}$. Both ML models perform reasonably well in all the control points, but slightly more evident discrepancies can be noticed at two locations, i.e. Building 1 and Water mark 3. The reason may be due to the relatively shallow depth and short flood duration in these two locations. In other words, the flood states at these two locations change rapidly during the flood event, thus providing insufficient training samples for the ML models to learn this highly dynamic 'feature'. 


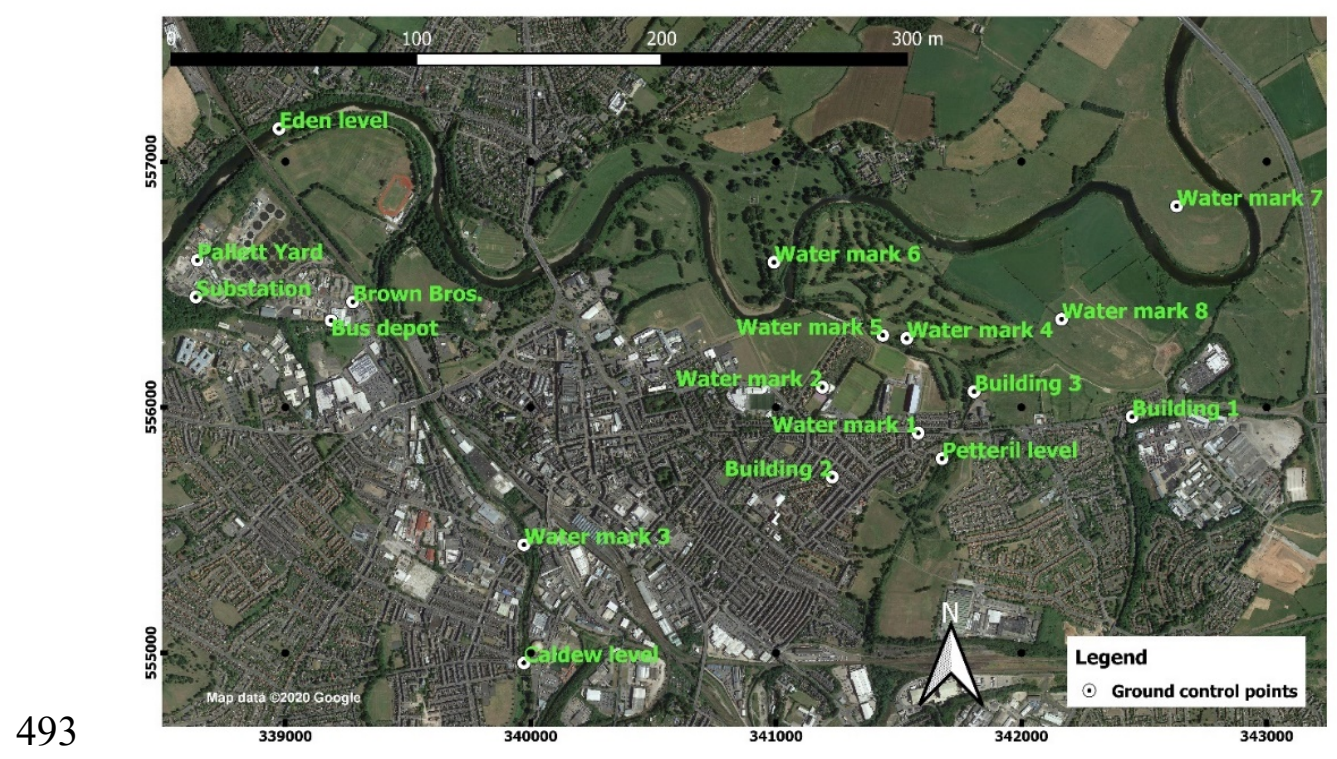

494 Figure 7. Control locations used for assessing model performance. 

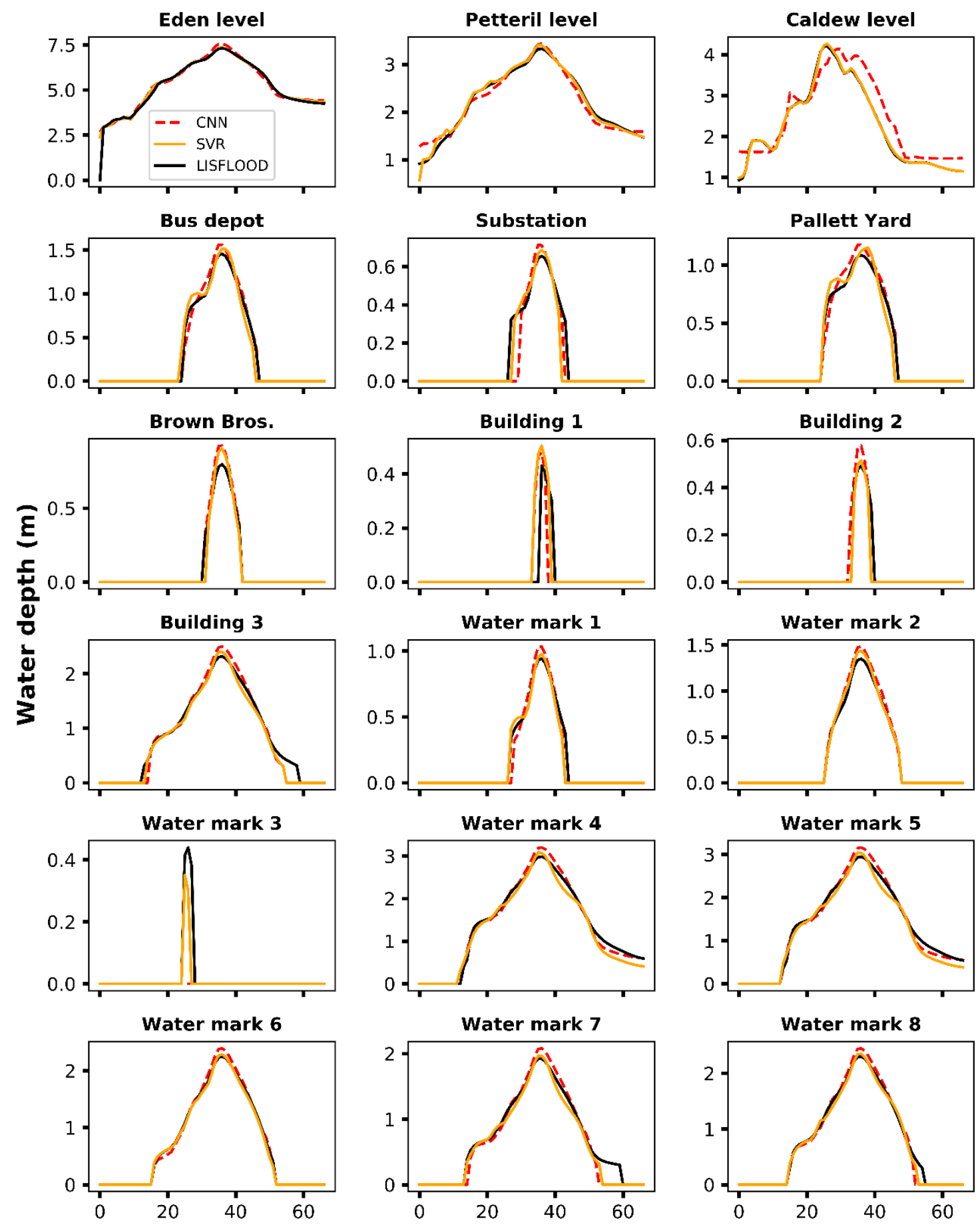

495
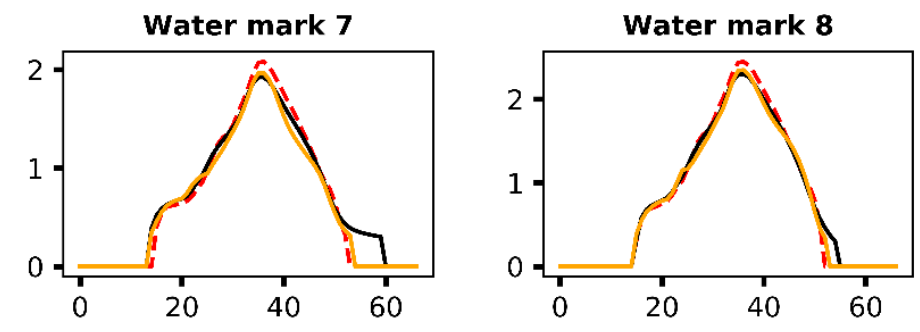

Time step (h)

496 Figure 8. Comparing the time-series of water depths predicted by CNN-2005 and the

497 SVR model against LISFLOOD-FP. 
501 calculated at the 18 control locations and the mean values are listed in Table 3 . Both of

502 the ML models perform reasonably well in this case, i.e. returning high mean values for

503 NSC and low mean values for RMSE. From these statistic matrices, the SVR model

504 seems to perform slightly better than CNN-2005. For example, the CNN model returns

505 a slightly lower mean NSE (0.86 vs. 0.91) than the SVR model. But after removing the

506 two control locations (Building 1 and Water mark 3) where the CNN model performs

507 less satisfactorily from the statistics, the performance of the two models is shown to be

508 both much improved and becomes similar (0.96 vs. 0.97 for CNN-2005 and SVR).

509 Arguably, these locations are exposed to small water depth and short flood duration and

510 therefore less important for overall flood risk management. As a whole, the satisfactory

511 point-wise comparison shows that the ML predictive models can aptly predict

512 maximum depths, arrival, and receding times. However, it is worth noting that the SVR

513 model was calibrated at these 18 control points and it is expected to perform better. The

514 SVR approach requires the use of 18 separately trained SVR models to make

515 predictions for these locations while a single CNN model $(\mathrm{CNN}-2005)$ is used and

516 sufficient to make comparable predictions, showing clear advantage in real-world

517 applications.

518 Table 3. The average NSEs and RMSEs calculated by the CNN-2005 and SVR models 519 at the 18 control locations.

\begin{tabular}{lcccc}
\hline & \multicolumn{2}{c}{ CNN-2005 } & \multicolumn{2}{c}{ SVR } \\
\hline Error measure & NSE & RMSE & NSE & RMSE \\
Average value & 0.86 & 0.11 & 0.91 & 0.08 \\
\hline
\end{tabular}


523 Fig. 9 presents four sets of predicted inundation maps at a time interval of 12 hours to represent the early, growing, peak and receding stages of the 2005 Carlisle flood event. The flood peak is predicted by all three models to occur at about 12:00 hours on $8^{\text {th }}$

526 January 2005, which is consistent with the actual event. From the results, it is evident

527 that the inundation maps produced by CNN-2005 are more effective in capturing the

528 inundated areas compared to the interpolated maps from the SVR models. The CNN2005 inundation maps are almost identical to those predicted by LISFLOOD-FP in all

530 four flood stages, suggesting that the model is able to accurately emulate the time-

531 varying water depths for the entire domain. Whilst the SVR model predicts a

532 satisfactory peak flood map in comparison to the other two models, it evidently

533 overestimates the inundated area for the flood growing and receding periods. In

534 addition, some unexpected small patches of water can be detected in the SVR flood

535 maps throughout the event. This is probably the numerical error caused by the RK

536 interpolation which considers surface elevation of the ground (i.e. bed elevation) as

537 auxiliary information (the correlation between water depth and surface elevation) and

538 may calculate incorrect depth values at those cells with low surface elevation, 539 irrespective of flooding conditions. 


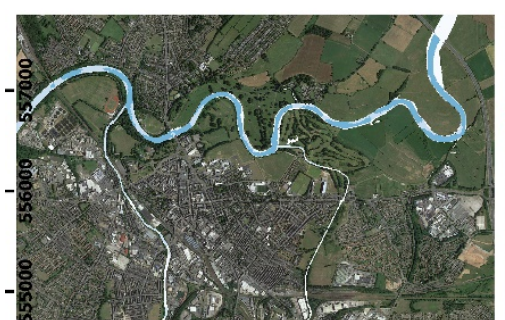

A. 12:00 hours 7 Jan 2005 - CNN

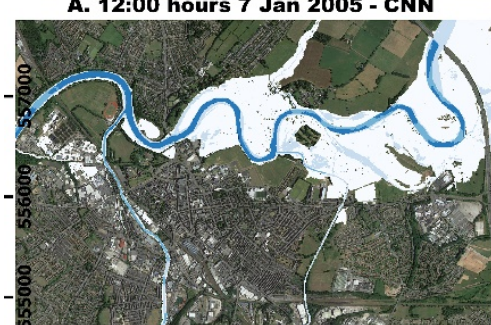

D. 00:00 hours 8 Jan 2005 - CNN

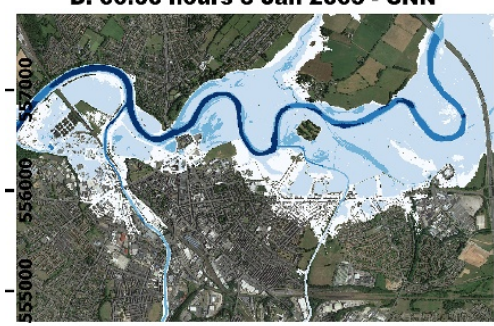

G. 12:00 hours 8 Jan 2005 - CNN

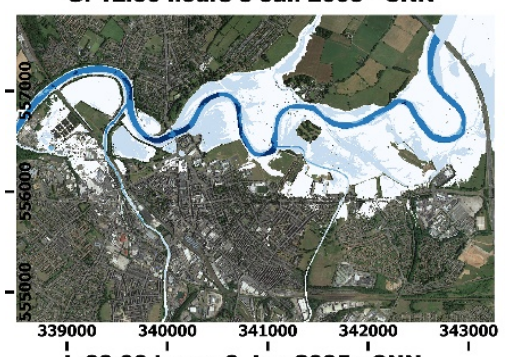

J. 00:00 hours 9 Jan 2005 - CNN

100

0

100

200

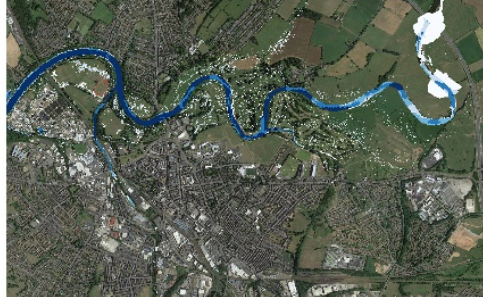

B. 12:00 hours 7 Jan 2005 - SVR

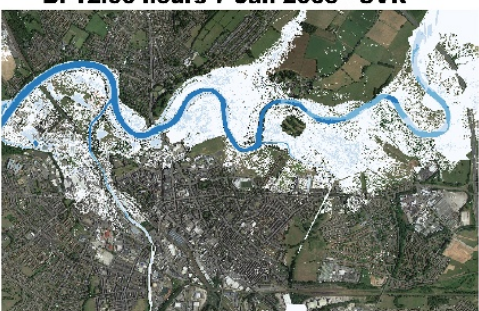

E. 00:00 hours 8 Jan 2005 - SVR

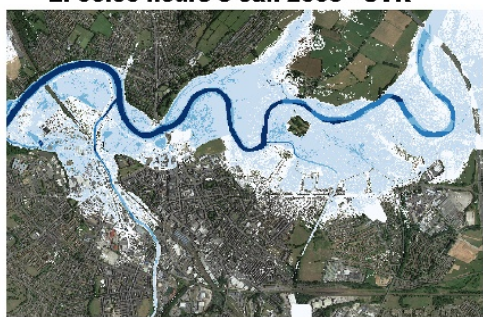

H. 12:00 hours 8 Jan 2005 - SVR

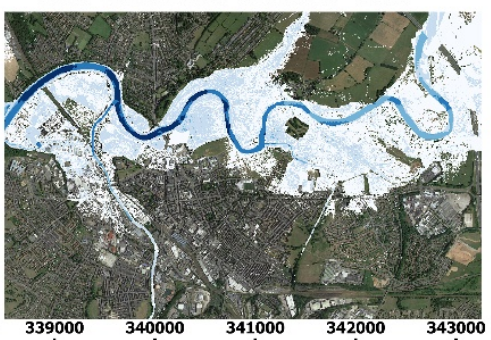

K. 00:00 hours 9 Jan 2005 - SVR

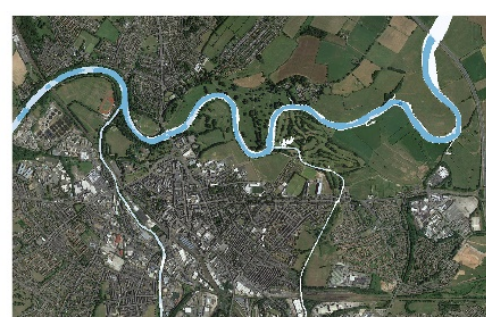

C. 12:00 hours 7 Jan 2005 - LF

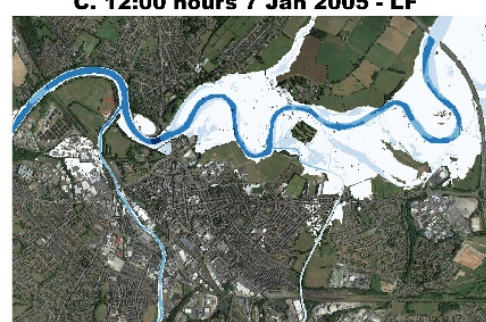

F. 00:00 hours 8 Jan 2005 - LF

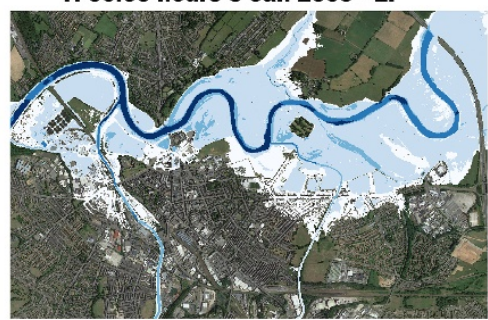

12:00 hours 8 Jan 2005 - LF

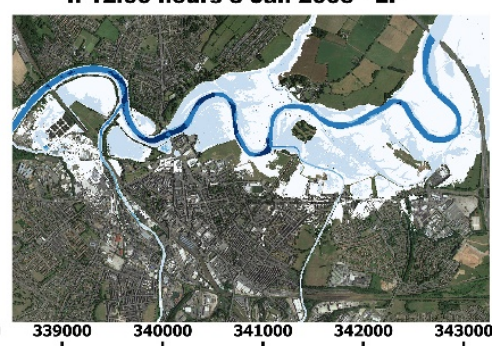

L. 00:00 hours 9 Jan 2005 - LF Water depth (m)

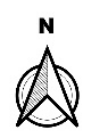

$0.3-1.6$

$1.6-3.2$

$3.2-4.8$

$4.8-6.4 \quad$ Map data $\odot 2020$ Google

541 Figure 9. The flood maps created from the CNN-2005, SVR and LISFLOOF-FP

predicted water depths during different stages of the flood event.

545 are presented in Table 4, showing that both of the CNN and SVR methods produce

546 results that are consistent with the LISFLOOD-FP outputs for all four flood stages. It is

547 interesting to note that although the CNN-2005 predictions visually appear to be much 
548 better than the SVR results (Fig. 9), this does not seem to be reflected in the descriptive 549 statistics.

550 Table 4. Descriptive statistics for the CNN-2005, SVR and LISFLOOD-FP predictions 551 at different stages of the event.

\begin{tabular}{lllll}
\hline Date & Model & Max (m) & Mean (m) & St. dev. (m) \\
\hline 12:00 & CNN-2005 & 4.71 & 0.11 & 0.57 \\
$7^{\text {th }}$ Jan 2005 & SVR & 4.28 & 0.11 & 0.57 \\
& LISFLOOD-FP & 4.95 & 0.12 & 0.62 \\
\hline $00: 00$ & CNN-2005 & 6.49 & 0.36 & 0.97 \\
$8^{\text {th }}$ Jan 2005 & SVR & 6.41 & 0.38 & 0.97 \\
& LISFLOOD-FP & 6.61 & 0.38 & 1.00 \\
\hline 12:00 & CNN-2005 & 8.17 & 0.85 & 1.47 \\
$8^{\text {th }}$ Jan 2005 & SVR & 8.11 & 0.81 & 1.42 \\
& LISFLOOD-FP & 8.10 & 0.81 & 1.44 \\
\hline $00: 00$ & CNN-2005 & 7.11 & 0.50 & 1.11 \\
$9^{\text {th }}$ Jan 2005 & SVR & 7.10 & 0.51 & 1.11 \\
& LISFLOOD-FP & 7.19 & 0.53 & 1.14 \\
\hline
\end{tabular}

552

To further investigate the robustness of the models, the spatial error maps (i.e.

554 the absolute difference of water depth between the ML and LISFLOOD-FP inundation

555 maps) for all four stages are produced and presented in Fig. 10, in which the first

556 column shows the error ranges from the CNN-2005 predictions and the second column

557 demonstrates the SVR error maps. From the error maps, it is clear that CNN-2005

558 produces flood maps matching much more closely with the reference LISFLOOD-FP

559 flood maps, with significantly smaller prediction errors. The resulting maximum error

$560(1.2 \mathrm{~m})$ of the $\mathrm{CNN}-2005$ model is almost at a similar level of the lowest error range of

561 the SVR interpolated maps of water depth. 

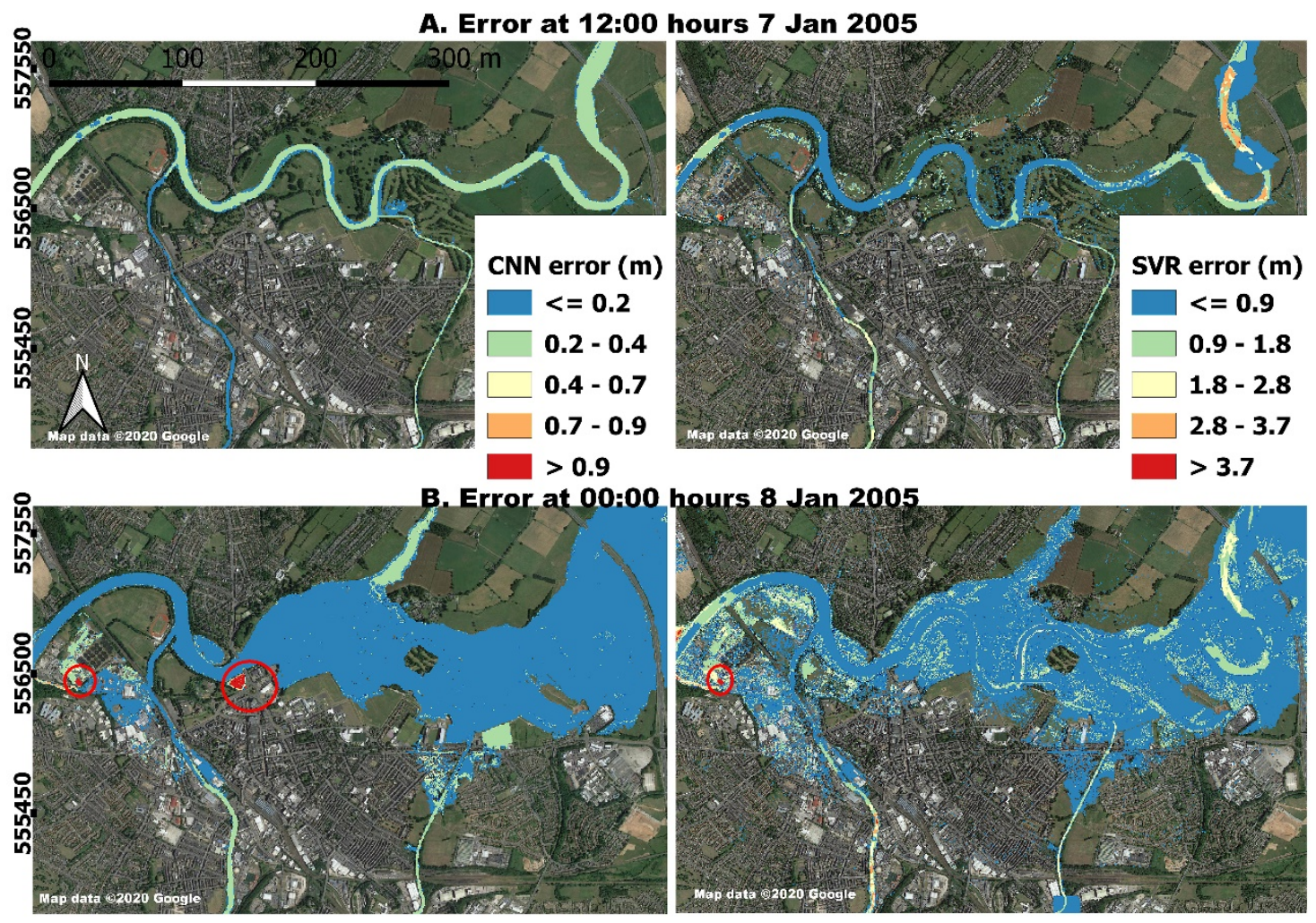

C. Error at 12:00 hours 8 Jan 2005

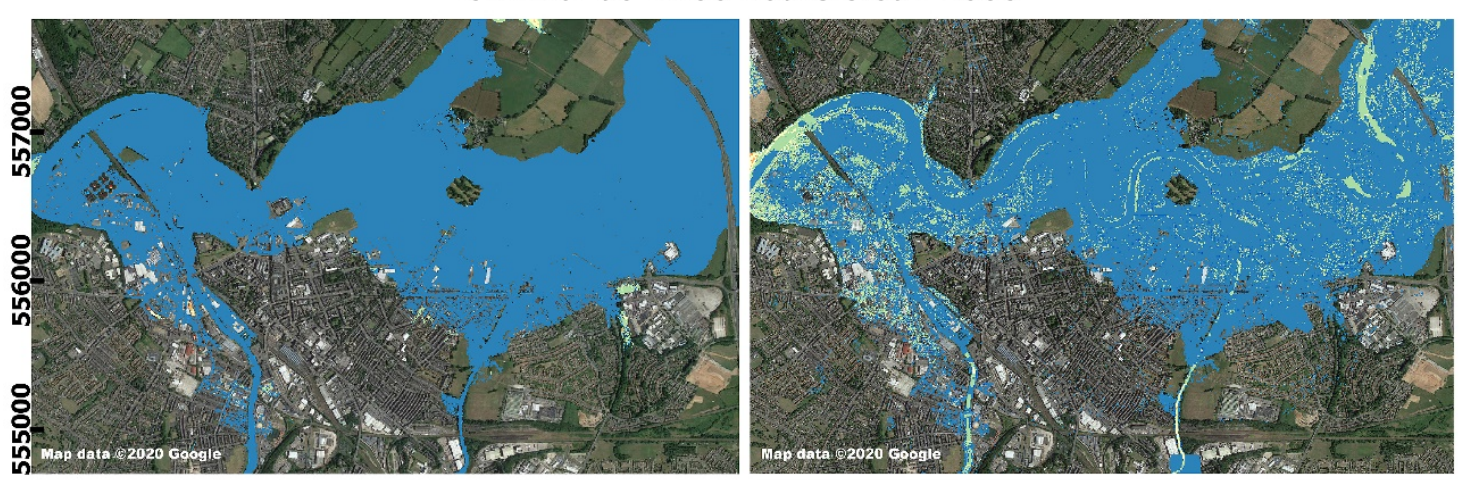

D. Error at 00:00 hours 9 Jan 2005
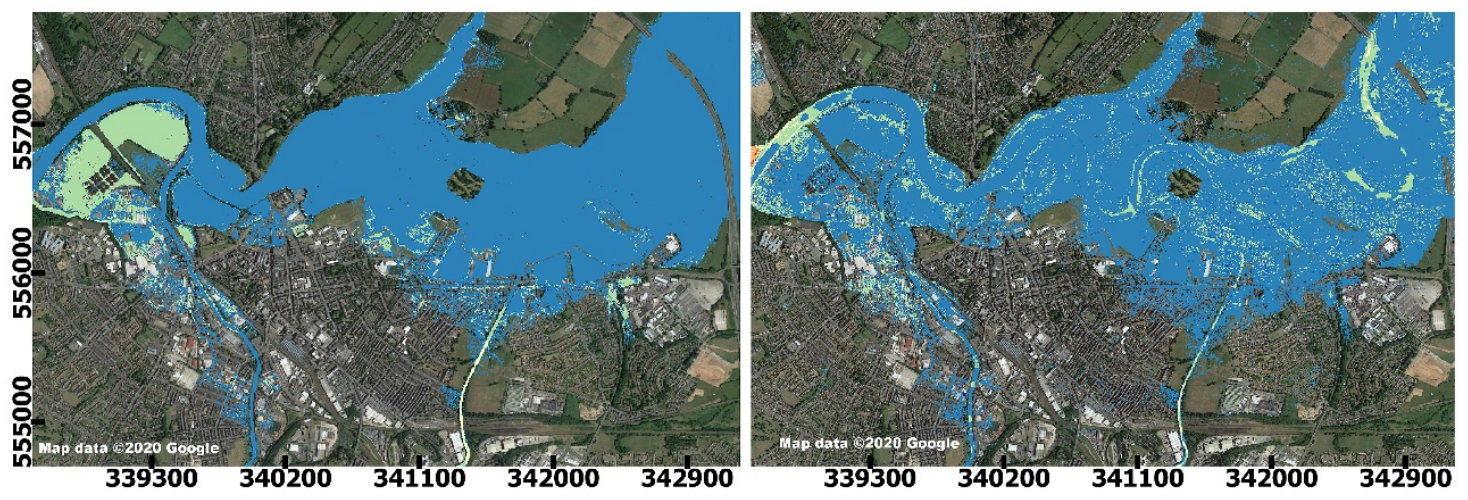

563 Figure 10. Spatial distribution of the model errors at the four flood stages, column 1:

564 CNN-2005 predictions; column 2: SVR results. 
565 During the peak flood stage (12:00 hours on $8^{\text {th }}$ January), the maximum error of the

$566 \mathrm{CNN}-2005$ prediction is $0.8 \mathrm{~m}$ and overall maximum prediction error of $1.2 \mathrm{~m}$ occurs at

$56700: 00$ hours on $8^{\text {th }}$ January. These big simulation errors are found to occur at the two

568 specific locations near to Hardwicke Circus (Centre) and Energi Trampoline Park

569 (West) (marked by red circles in Fig. 10). To further investigate the performance of the

570 models after removing these singular points from the statistics, the $99^{\text {th }}$ percentile

571 prediction error during the peak is calculated for CNN-2005, which is $0.2 \mathrm{~m}$. This means

572 that $99 \%$ of the cells inside the simulation domain have an error less or equal to $0.2 \mathrm{~m}$

573 when predicting water depth at the peak flood stage. Similarly, the $99^{\text {th }}$ percentile error

574 of CNN-2005 for the flood growing and receding periods is $0.4 \mathrm{~m}$. However, the $99^{\text {th }}$

575 percentile errors of the SVR model in predicting the water depth at the peak, growing

576 and receding periods are $1.6 \mathrm{~m}, 1.9 \mathrm{~m}$ and $1.5 \mathrm{~m}$, respectively. The small prediction errors

577 of CNN-2005 in all flood stages confirm that the model is more effective in correctly

578 predicting the spatially varying water depths.

579 To continue on quantifying the performance of the ML predictive models in

580 reproducing the spatial predictions of LISFLOOD-FP, the flood extents are derived for

581 different stages of the flood event and the number of cells being correctly classified as

582 flooded is counted to calculate the recall, precision and F1 scores, which are

583 summarised in Table 5. Herein, a threshold value of $0.3 \mathrm{~m}$ is used to delineate wet-dry

584 cells. From the statistic scores, it is clear that almost all of the cells (nearly 100\%)

585 classified by the LISFLOOD-FP as flooded are correctly captured by CNN-2005. As a

586 summary, CNN-2005 emulates the outputs from LISFLOOD-FP to a high level of

587 accuracy throughout the whole flood event and outperforms the SVR model to a large

588 margin. It may be concluded that, whilst the results predicted by the SVR model may be

589 of higher accuracy at sampled points/cells (Sect. 4.1), the model's accuracy is 
590 drastically reduced when interpolation is conducted to produce the spatially distributed

591 depth maps. Whilst it may be possible to enhance the accuracy of the SVR model by

592 increasing the number of samples, this will subsequently increase the number of SVR

593 models that need to be trained and therefore require more computational resources. On

594 the other hand, the CNN model predicts spatially varying water depth at a much higher

595 level of accuracy and a single model is sufficient for city-scale inundation modelling,

596 proving great potential for real-world applications.

597 Table 5. Spatial accuracy scores of the CNN-2005 and SVR models against

598 LISFLOOD-FP during the flood initiation, growing, peak and recession stages.

\begin{tabular}{ccccccc}
\hline Date & \multicolumn{2}{c}{ Precision } & \multicolumn{2}{c}{ Recall } & & F1 \\
& CNN- & SVR & CNN- & SVR & CNN- & SVR \\
& $\mathbf{2 0 0 5}$ & & $\mathbf{2 0 0 5}$ & & $\mathbf{2 0 0 5}$ & \\
\hline 12:00 $7^{\text {th }}$ Jan 2005 & 0.99 & 0.59 & 0.96 & 0.74 & 0.98 & 0.65 \\
00:00 $8^{\text {th }}$ Jan 2005 & 0.98 & 0.75 & 0.97 & 0.87 & 0.98 & 0.81 \\
12:00 $8^{\text {th }}$ Jan 2005 & 0.98 & 0.90 & 0.99 & 0.97 & 0.99 & 0.93 \\
00:00 9 $9^{\text {th }}$ Jan 2005 & 0.99 & 0.88 & 0.96 & 0.94 & 0.98 & 0.90 \\
\hline
\end{tabular}

599

600

601

602

603

604

605

606

607

608

Finally, the CNN-2005 predicted peak flood extent at 12:00 hours on January $8^{\text {th }}$ is overlaid with the extents derived from the LISFLOOD-FP outputs and also EA postevent survey in Fig. 11. Overall, the CNN-2005 flood extent matches well with that produced by LISFLOOD-FP, which is as expected since a high classification accuracy between the two sets of model outputs (Table 5). But slight discrepancies can be seen between the CNN-2005 and the surveyed flood extents near to Denton Holme (close to Upstream 3), Melbourne Park (left bank of River Petteril), Willow Holme (confluence of River Caldew and Little Caldew) and Warwick Road East and West. This may be attributed to the assumptions we made in upstream boundary conditions (the use of 
610 flood simulations). But it should be noted that the CNN model is trained to emulate the

611 LISFLODD-FP results in this work, which is deemed to be highly success at this sense.

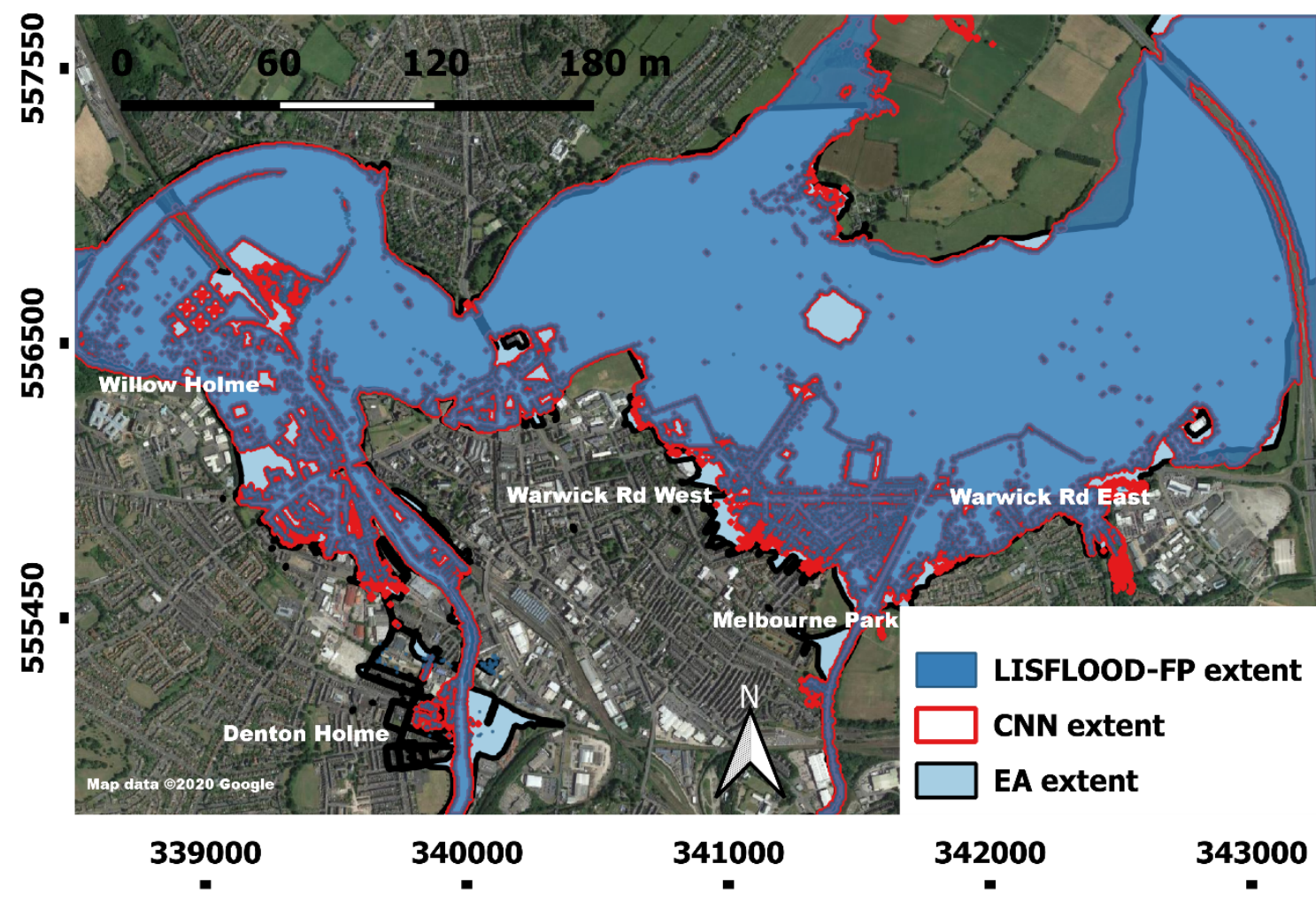

613 Figure 11. Comparing the CNN-2005 derived flood extent with the LISFLOOD-FP and 614 surveyed extents.

\subsection{Comparison between CNN-2015 and LISFLOOD-FP for the 2015 event}

616 It has been demonstrated that the CNN model is able to emulate the LISFLOOD

617 predictions to a high level of accuracy for the 2005 Carlisle flood and the model

618 outperforms the SVR method in predicting spatially distributed water depths. In this

619 section, we further confirm the effectiveness of the CNN model as an emulator of a

620 hydraulic model for more complex flood dynamics that is influenced by flood defences

621 (i.e. changes of domain topography). The magnitude of the 2015 flood event exceeded

622 the design criteria of flood walls and embankments constructed after the 2005 event. 
623 But the existence of the flood defence system might effectively delay the arrival of the

624 flood and leave more time for the emergency responders to take necessary actions to

625 mitigate the potential impact (Cumbria City Council, 2017). Herein, we conduct

626 numerical experiments to examine the ability of the CNN model (i.e. CNN-2015), after

627 being trained, in capturing the change of flood dynamics as induced by the topographic 628 changes.

629 Fig. 12 presents the flood maps produced by the trained CNN-2015 model for

630 the early, growing, peak and receding stages of the event. From the results, the impact

631 of the flood defences can be clearly seen during the early and growing periods of the

632 event (i.e. 10:00 hours on $5^{\text {th }}$ December and 18:00 hours on $5^{\text {th }}$ December, respectively).

633 The flood water is held back by the defences at these early stages, leading to a delay in

634 inundation, which has been successfully captured by CNN-2015. Once the flood

635 defences are overtopped, severe inundation occurs and evolves rapidly, which is again

636 reliably predicted by both of the CNN-2015 and LISFLOOD-FP models. Quantitively,

637 the mean NSE and RMSE calculated against the time series of water depth at the control

638 points are respectively 0.94 and $0.18 \mathrm{~m}$, confirming CNN-2015 successfully emulates

639 the outputs of LISFLOOD-FP to a very high level of accuracy.

640 Fig. 13 shows the error maps of the $\mathrm{CNN}-2015$ predictions against the

641 LISFLOOD-FP outputs for all four flood stages. Similar to the CNN-2005 results, the

642 maximum error $(2.2 \mathrm{~m})$ occurs during the flood growing stage at the same locations (i.e.

643 near to Hardwicke Circus and Energi Trampoline Park) and the maximum prediction

644 error during the peak (06:00 hours $6^{\text {th }}$ December) is estimated to be $0.8 \mathrm{~m}$. The $99^{\text {th }}$

645 percentile errors calculated for the early, growing, peak and receding periods are $0.5 \mathrm{~m}$,

$6461 \mathrm{~m}, 0.5 \mathrm{~m}$ and $0.3 \mathrm{~m}$, respectively. Table 6 presents the recall, precision and $F 1$ scores 
647 for the simulation, confirming that $\mathrm{CNN}-2015$ is highly accurate in correctly classifying

648 the wet/dry cells at all stages of the event.
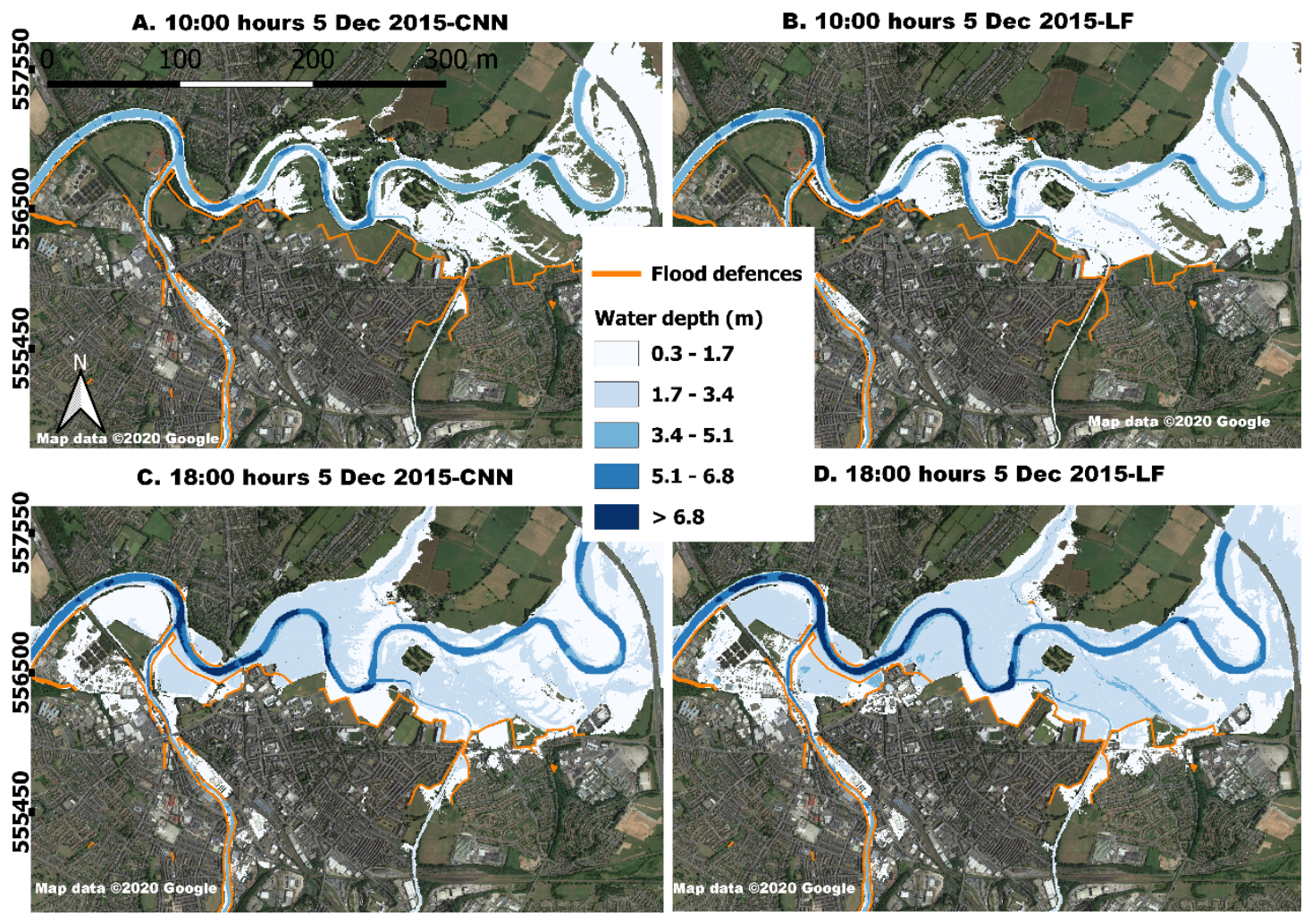

5.1 - 6.8 D. 18:00 hours 5 Dec 2015-LF

E. 06:00 hours 6 Dec 2015-CNN

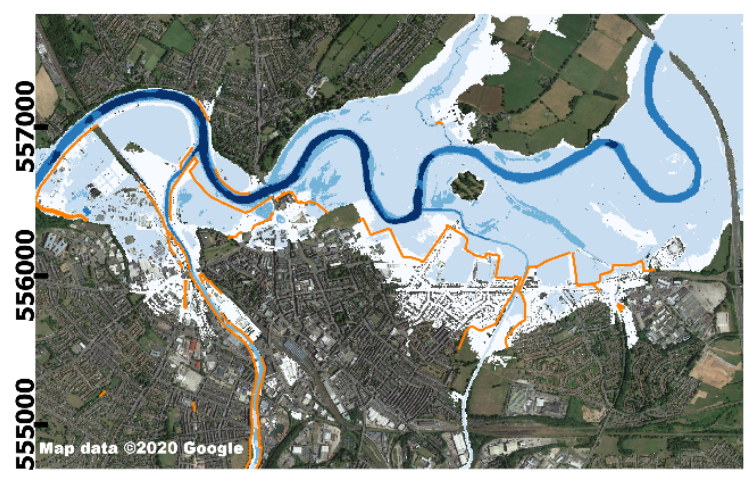

G. 06:00 hours 7 Dec 2015-CNN

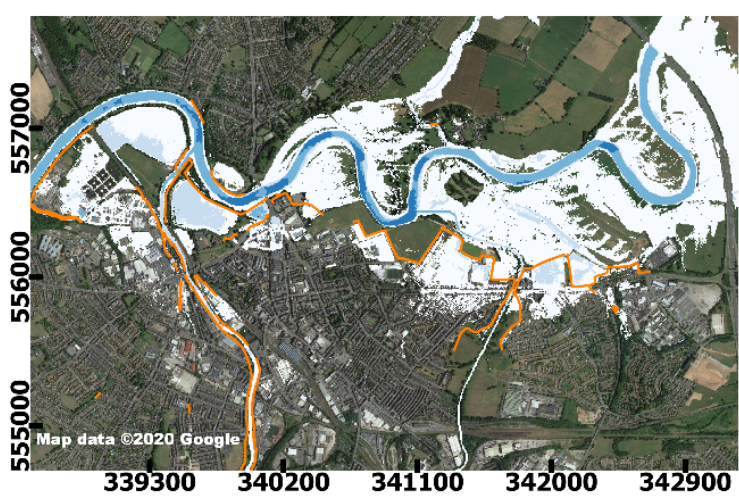

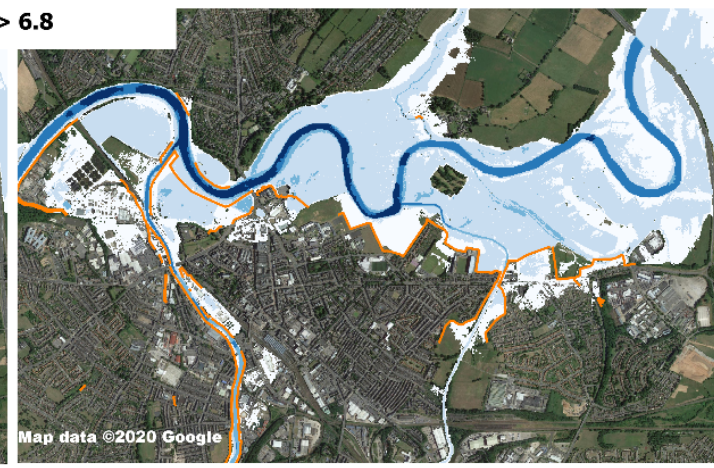

F. 06:00 hours 6 Dec 2015-LF

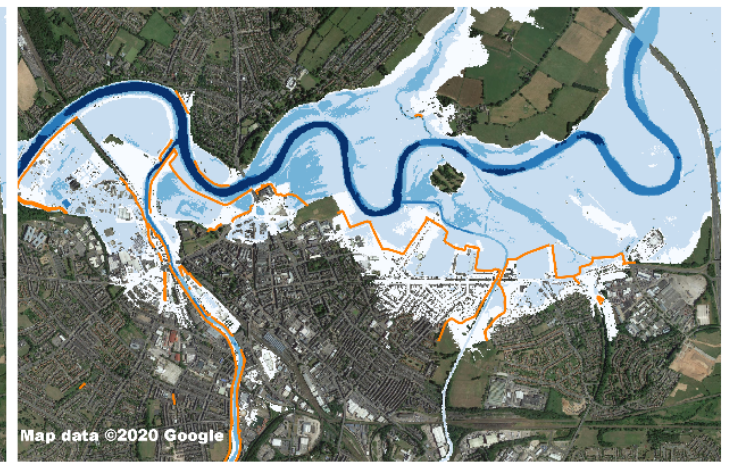

H. 06:00 hours 7 Dec 2015-LF

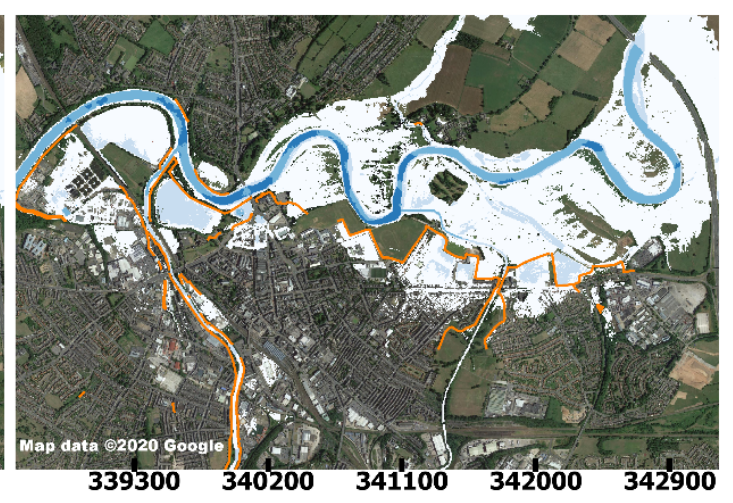


650 Figure 12. The CNN-2015 and LISFLOOD-FP predicted flood maps, revealing the

651 influence of the flood defences at different stages of the 2015 flood event on $5^{\text {th }}$ and $6^{\text {th }}$

652 December.

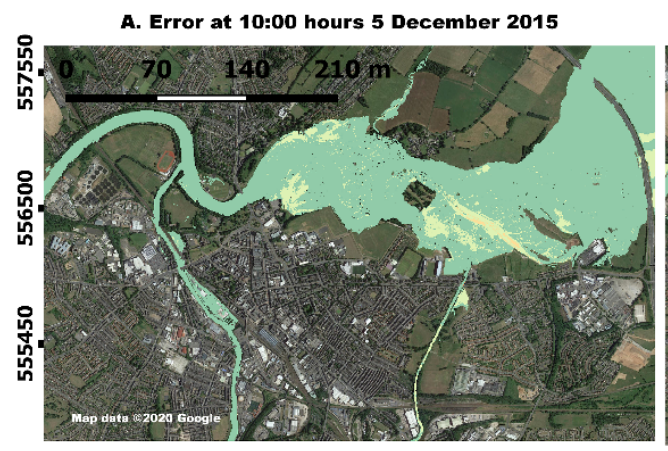

C. Error at 06:00 hours 6 December 2015

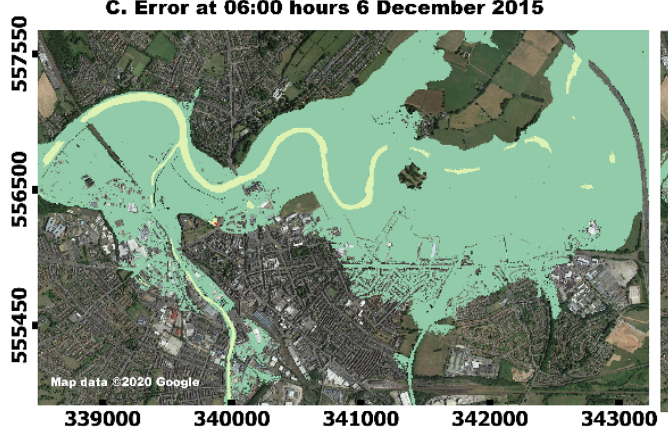

B. Error at 18:00 hours 5 December 2015

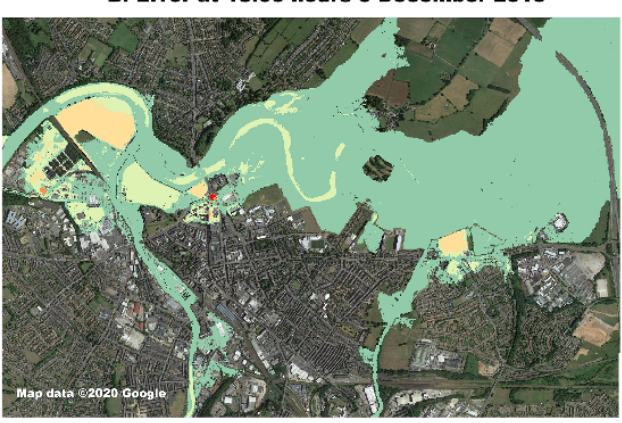

D. Error at 06:00 hours 7 December 2015

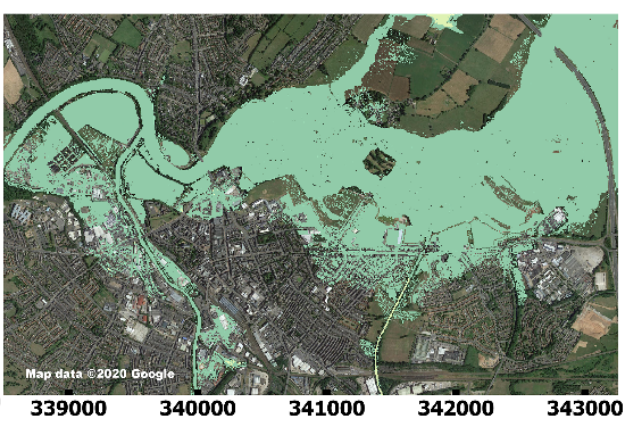

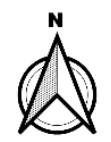

Depth error (m) $<=0.4$

654 Figure 13. Spatial distribution of the CNN-2015 model errors at the four flood stages.

Table 6. Classification accuracy scores of CNN-2015 against LISFLOOD-FP in

656 predicting the 2015 flood event.

\begin{tabular}{lccc}
\hline Date & Precision & Recall & F1 \\
\hline 10:00 5 ${ }^{\text {th }}$ Dec 2015 & 0.99 & 0.89 & 0.94 \\
18:00 $5^{\text {th }}$ Dec 2015 & 0.99 & 0.94 & 0.97 \\
$06: 006^{\text {th }}$ Dec 2015 & 0.99 & 0.99 & 0.99 \\
$06: 008^{\text {th }}$ Dec 2015 & 0.99 & 0.93 & 0.96 \\
\hline
\end{tabular}

659 intervening the flood dynamics, the four-stage inundation maps from the simulation

660 without considering the defences are produced and presented in Fig. 14. Comparing

661 with flood maps in Fig. 12 where flood defences are embedded in the simulation, it can 
664 defences is detected during the flood receding stage, where the water is held back by the 665 defences in the floodplain for a longer duration. At the flood peak, the inundation maps 666 predicted by the two models (with and without considering defences) become more 667 consistent, which is expected.
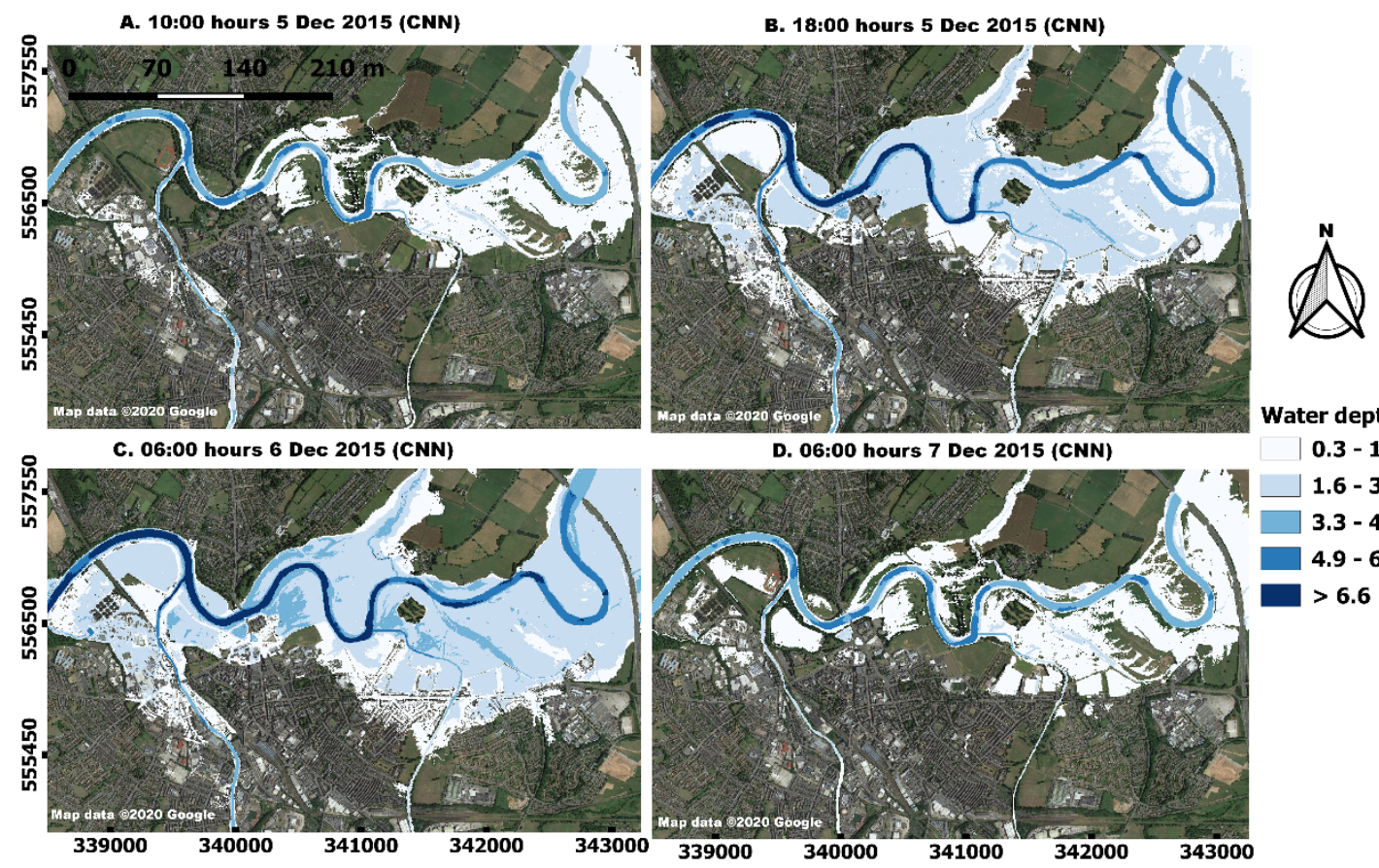

Water depth (m) $0.3-1.6$

669 Figure 14. The flood maps predicted by the CNN model for the 2015 event without 670 considering flood defences.

\subsection{Computational time}

672 Table 7 provides the run times required by CNN-2005 and LISFLOOD-FP to reproduce the 2005 Carlisle flood. For the CNN model, it takes only a couple of minutes to

674 generate the sequential water depth maps for the entire domain and a few minutes to

675 train the model. It should be noted that, once trained, the CNN model can be used to

676 efficiently predict different flood scenarios. Drawing a completely fair comparison

677 between the two modelling approaches is difficult since they use different computing

678 platforms. Nevertheless, the numbers in Table 7 can still indicate that the CNN model is 
679 computationally efficient for real-time applications and can potentially be further

680 explored for supporting probabilistic flood forecasts using ensemble numerical weather 681 predications.

682 Table 7. The runtimes of the CNN-2005 and LISFLOOD-FP models for simulating the 6832005 Carlisle flood event.

\begin{tabular}{ccccc}
\hline Model & $\begin{array}{c}\text { Train } \\
\text { time }\end{array}$ & $\begin{array}{c}\text { Total time required to } \\
\text { simulate entire event }\end{array}$ & $\begin{array}{c}\text { Output } \\
\text { format }\end{array}$ & Computing device \\
\hline CNN & 3 minutes & 2 minutes & Raster & $\begin{array}{c}\text { NVIDIA Tesla P100 } \\
\text { GPU }\end{array}$ \\
& & & & Intel I5-9400 \\
LISFLOOD- & - & 77 minutes & Raster & $2.90 \mathrm{GHz} \mathrm{CPU}$ \\
FP & & & &
\end{tabular}

684

\section{Conclusions}

686 The need for evidence-based flood management is greater than ever before due to rapid

687 urbanisation and climate change that have already led to increased flood risk across the world. In this context, fast, reliable and robust modelling tools for real-time flood prediction/forecasting is important for assessing the multidimensional social and economic impacts of and providing reliable forecasts to enhance societal resilience to

691 flooding. This work introduces a deep CNN approach for rapid fluvial flood modelling

692 that can potentially be leveraged for operational flood nowcasting or forecasting. The

693 idea underpinning the proposed study is that cell-based water depths in a floodplain are

694 a function of time varying discharge and the time of observation at the upstream.

695 Therefore, a non-linear function can be fitted between sequences of historical

696 geographically distributed water levels/depths and observed discharges to predict water 697 levels for the future flood events. The results show that the CNN model can effectively 
emulate the outputs (i.e. water depths) of a 2D hydraulic model to a high accuracy. It

699 also shows that the model can be easily re-trained to account for the major

700

701

702

703

704

705

706

707

708

709

710

711

712

713

714

715

716

717

718

719

720

721

722

723

724 topographical change (e.g. construction of flood defences) and capture the resulting influence on flood dynamics. Compared with traditional ML methods such as SVM, a single CNN model has the capability to make predictions for a domain consisting hundreds of thousands of cells. Due to the high computational efficiency, superior performance and simplicity, the proposed method offers a promising tool for real-time nowcasting/forecasting of flood inundation.

\section{Model and data availability}

The models constructed in this paper and reproduceable data have been made available through https://github.com/SRKabir/Rapid_FloodModelling_CNN.

\section{Acknowledgements}

This work is partly funded by the Newton Fund and UK Met Office 'WCSSP-India Lot 7: Building a Flood Hazard Impact Model for India (FHIM-India) (DN394978)’ project.

\section{References}

Abdeljaber, O., Avci, O., Kiranyaz, M. S., Boashash, B., Sodano, H. \& Inman, D. J. (2018) 1-D CNNs for structural damage detection: Verification on a structural health monitoring benchmark data. Neurocomputing 275, 1308-1317. Elsevier. doi:10.1016/J.NEUCOM.2017.09.069

Aldridge, T., Gunawan, O., Moore, R. J., Cole, S. J. \& Price, D. (2016) A surface water flooding impact library for flood risk assessment. (M. Lang, F. Klijn \& P. Samuels, Eds.)E3S Web Conf. 7, 18006. doi:10.1051/e3sconf/20160718006

Allen, D., Newell, A. \& Butcher, A. (2010) Preliminary review of the geology and hydrogeology of the Eden DTC sub-catchments. Br. Geol. Surv. Open Report, 
726

727

728

729

730

731

732

733

734

735

736

737

738

739

740

741

742

743

744

745

746

747

748

749

750

751

752

753

754

755

Almeida, G. A. M. de, Bates, P., Freer, J. E. \& Souvignet, M. (2012) Improving the stability of a simple formulation of the shallow water equations for 2-D flood modeling. Water Resour. Res. 48(5). John Wiley \& Sons, Ltd. doi:10.1029/2011WR011570

Amarnath, G., Umer, Y. M., Alahacoon, N. \& Inada, Y. (2015) Modelling the flood-risk extent using LISFLOOD-FP in a complex watershed: case study of Mundeni Aru River Basin, Sri Lanka. Proc. IAHS 370, 131-138. Copernicus Publications. doi:10.5194/piahs-370-131-2015

Barnston, A. G. (1992) Correspondence among the Correlation, RMSE, and Heidke Forecast Verification Measures; Refinement of the Heidke Score. Weather Forecast. 7(4), 699-709. doi:10.1175/15200434(1992)007<0699:CATCRA $>2.0 . C O ; 2$

Bates, P.D \& Roo, A. P. . De. (2000) A simple raster-based model for flood inundation simulation. J. Hydrol. 236(1-2), 54-77. Elsevier. doi:10.1016/S00221694(00)00278-X

Bates, Paul D., Horritt, M. S. \& Fewtrell, T. J. (2010) A simple inertial formulation of the shallow water equations for efficient two-dimensional flood inundation modelling. J. Hydrol. 387(1-2), 33-45. Elsevier. doi:10.1016/J.JHYDROL.2010.03.027

Berkhahn, S., Fuchs, L. \& Neuweiler, I. (2019) An ensemble neural network model for real-time prediction of urban floods. J. Hydrol. 575, 743-754. Elsevier. doi:10.1016/J.JHYDROL.2019.05.066

Bermúdez, M., Cea, L. \& Puertas, J. (2019) A rapid flood inundation model for hazard mapping based on least squares support vector machine regression. J. Flood Risk Manag. 12(S1), e12522. John Wiley \& Sons, Ltd. doi:10.1111/jfr3.12522

Bhola, P., Leandro, J. \& Disse, M. (2018) Framework for Offline Flood Inundation Forecasts for Two-Dimensional Hydrodynamic Models. Geosciences 8(9), 346. Multidisciplinary Digital Publishing Institute. doi:10.3390/geosciences8090346

Chang, L.-C., Amin, M., Yang, S.-N. \& Chang, F.-J. (2018) Building ANN-Based Regional Multi-Step-Ahead Flood Inundation Forecast Models. Water 10(9), 1283. 
Chang, L.-C., Chang, F.-J., Yang, S.-N., Kao, I.-F., Ku, Y.-Y., Kuo, C.-L. \& Amin, I. (2018) Building an Intelligent Hydroinformatics Integration Platform for Regional Flood Inundation Warning Systems. Water 11(1), 9. Multidisciplinary Digital Publishing Institute. doi:10.3390/w11010009

Chang, L.-C., Shen, H.-Y. \& Chang, F.-J. (2014) Regional flood inundation nowcast using hybrid SOM and dynamic neural networks. J. Hydrol. 519, 476-489. Elsevier. doi:10.1016/J.JHYDROL.2014.07.036

Chang, L.-C., Shen, H.-Y., Wang, Y.-F., Huang, J.-Y. \& Lin, Y.-T. (2010) Clusteringbased hybrid inundation model for forecasting flood inundation depths. J. Hydrol. 385(1-4), 257-268. Elsevier. doi:10.1016/J.JHYDROL.2010.02.028

Cortes, C. \& Vapnik, V. (1995) Support-vector networks. Mach. Learn. 20(3), 273-297. doi:10.1007/BF00994018

Council, C. C. (2017) Carlisle Flood Investigation Report Final V1. Available in https://www.cumbria.gov.uk/planningenvironment/flooding/flood investigation_reports_carlisle.asp. Accessed $07 / 08 / 2020$

Hengl, T., Heuvelink, G. B. M. \& Rossiter, D. G. (2007) About regression-kriging: From equations to case studies. Comput. Geosci. 33(10), 1301-1315. doi:https://doi.org/10.1016/j.cageo.2007.05.001

Ioffe, S. \& Szegedy, C. (2015, February) Batch Normalization: Accelerating Deep Network Training by Reducing Internal Covariate Shift. arXiv e-prints.

Jhong, Y.-D., Chen, C.-S., Lin, H.-P. \& Chen, S.-T. (2018) Physical Hybrid Neural Network Model to Forecast Typhoon Floods. Water 10(5), 632. Multidisciplinary Digital Publishing Institute. doi:10.3390/w10050632

Kabir, S., Patidar, S. \& Pender, G. (2020) A Machine Learning Approach for Forecasting and Visualizing Flood Inundation Information. Proc. Inst. Civ. Eng. Water Manag. 1-29. ICE Publishing. doi:10.1680/jwama.20.00002

Kiranyaz, S., Avci, O., Abdeljaber, O., Ince, T., Gabbouj, M. \& Inman, D. J. (2019) 1D 786 Convolutional Neural Networks and Applications: A Survey. Retrieved from http://arxiv.org/abs/1905.03554 
Kiranyaz, S., Ince, T., Hamila, R. \& Gabbouj, M. (2015) Convolutional Neural Networks for patient-specific ECG classification. Conf. Proc. ... Annu. Int. Conf. IEEE Eng. Med. Biol. Soc. IEEE Eng. Med. Biol. Soc. Annu. Conf. 2015, 26082611. United States. doi:10.1109/EMBC.2015.7318926

Knijff, J. M. Van Der, Younis, J. \& Roo, A. P. J. De. (2010) LISFLOOD: a GIS-based distributed model for river basin scale water balance and flood simulation. Int. J. Geogr. Inf. Sci. 24(2), 189-212. Taylor \& Francis. doi:10.1080/13658810802549154

Komi, K., Neal, J., Trigg, M. A. \& Diekkrüger, B. (2017) Modelling of flood hazard extent in data sparse areas: a case study of the Oti River basin, West Africa. $J$. Hydrol. Reg. Stud. 10, 122-132. doi:https://doi.org/10.1016/j.ejrh.2017.03.001

Leedal, D., Neal, J., Beven, K., Young, P. \& Bates, P. (2010) Visualization approaches for communicating real-time flood forecasting level and inundation information. $J$. Flood Risk Manag. 3(2), 140-150. John Wiley \& Sons, Ltd. doi:10.1111/j.1753318X.2010.01063.X

Lin, G.-F., Lin, H.-Y. \& Chou, Y.-C. (2013) Development of a real-time regionalinundation forecasting model for the inundation warning system. $J$. Hydroinformatics 15(4), 1391-1407. doi:10.2166/hydro.2013.202

Liu, Y. \& Pender, G. (2015) A flood inundation modelling using v-support vector machine regression model. Eng. Appl. Artif. Intell. 46, 223-231. Pergamon. doi:10.1016/J.ENGAPPAI.2015.09.014

Ming, X., Liang, Q., Xia, X., Li, D. \& Fowler, H. J. (2020) Real-Time Flood Forecasting Based on a High-Performance 2-D Hydrodynamic Model and Numerical Weather Predictions. Water Resour. Res. 56(7), e2019WR025583. John Wiley \& Sons, Ltd. doi:10.1029/2019WR025583

Munandar, D., Arisal, A., Riswantini, D. \& Rozie, A. F. (2018) Text Classification for Sentiment Prediction of Social Media Dataset using Multichannel Convolution Neural Network. 2018 Int. Conf. Comput. Control. Informatics its Appl., 104-109. doi:10.1109/IC3INA.2018.8629522

Nash, J. E. \& Sutcliffe, J. V. (1970) River flow forecasting through conceptual models part I - A discussion of principles. J. Hydrol. 10(3), 282-290. Elsevier. 

doi:10.1016/0022-1694(70)90255-6

819 Neal, J., Dunne, T., Sampson, C., Smith, A. \& Bates, P. (2018) Optimisation of the two820 dimensional hydraulic model LISFOOD-FP for CPU architecture. Environ. Model. Softw. 107, 148-157. doi:https://doi.org/10.1016/j.envsoft.2018.05.011

Neal, J., Keef, C., Bates, P., Beven, K. \& Leedal, D. (2013) Probabilistic flood risk mapping including spatial dependence. Hydrol. Process. 27(9), 1349-1363. John Wiley \& Sons, Ltd. doi:10.1002/hyp.9572

Paiva, R. C. D. de, Buarque, D. C., Collischonn, W., Bonnet, M.-P., Frappart, F., Calmant, S. \& Bulhões Mendes, C. A. (2013) Large-scale hydrologic and hydrodynamic modeling of the Amazon River basin. Water Resour. Res. 49(3), 1226-1243. John Wiley \& Sons, Ltd. doi:10.1002/wrcr.20067

Parkes, B. L., Cloke, H. L., Pappenberger, F., Neal, J. \& Demeritt, D. (2013) Reducing Inconsistencies in Point Observations of Maximum Flood Inundation Level. Earth Interact. 17(6), 1-27. doi:10.1175/2012EI000475.1

Raghavendra. N, S. \& Deka, P. C. (2014) Support vector machine applications in the field of hydrology: A review. Appl. Soft Comput. 19, 372-386. doi:https://doi.org/10.1016/j.asoc.2014.02.002

Roberts, N. M., Cole, S. J., Forbes, R. M., Moore, R. J. \& Boswell, D. (2009) Use of high-resolution NWP rainfall and river flow forecasts for advance warning of the Carlisle flood, north-west England. Meteorol. Appl. 16(1), 23-34. John Wiley \& Sons, Ltd. doi:10.1002/met.94

Sanders, B. F. \& Schubert, J. E. (2019) PRIMo: Parallel raster inundation model. $A d v$. Water Resour. 126, 79-95. doi:https://doi.org/10.1016/j.advwatres.2019.02.007

Shen, H.-Y. \& Chang, L.-C. (2013) Online multistep-ahead inundation depth forecasts by recurrent NARX networks. Hydrol. Earth Syst. Sci. 17(3), 935-945. Copernicus Publications. doi:10.5194/hess-17-935-2013 
Learn. Res. 15(56), 1929-1958. Retrieved from http://jmlr.org/papers/v15/srivastava14a.html

851 Xia, X., Liang, Q. \& Ming, X. (2019) A full-scale fluvial flood modelling framework 852 based on a high-performance integrated hydrodynamic modelling system (HiPIMS). Adv. Water Resour. 132, 103392. Elsevier. doi:10.1016/J.ADVWATRES.2019.103392

855 Yamazaki, D., Kanae, S., Kim, H. \& Oki, T. (2011) A physically based description of 856 floodplain inundation dynamics in a global river routing model. Water Resour.

857 Res. 47(4). John Wiley \& Sons, Ltd. doi:10.1029/2010WR009726

858 Zihlmann, M., Perekrestenko, D. \& Tschannen, M. (2017) Convolutional Recurrent

859 Neural Networks for Electrocardiogram Classification. Retrieved from

860 http://arxiv.org/abs/1710.06122

861 